This article has been accepted for publication in MNRAS (c) 2018 The Authors. Published by Oxford University Press on behalf of the Royal Astronomical Society. All rights reserved. 


\title{
Lyman $\alpha$ forest and non-linear structure characterization in Fuzzy Dark Matter cosmologies
}

\author{
Matteo Nori ${ }^{\oplus}, 1,2,3 \star$ Riccardo Murgia,,${ }^{4,5}$ Vid Iršič, ${ }^{6}$ Marco Baldi ${ }^{\oplus 1,2,3}$ and \\ Matteo Viel ${ }^{4,5,7}$ \\ ${ }^{1}$ Dipartimento di Fisica e Astronomia, Alma Mater Studiorum - University of Bologna, Via Piero Gobetti 93/2, I-40129 Bologna BO, Italy \\ ${ }^{2}$ INAF - Osservatorio Astronomico di Bologna, Via Piero Gobetti 93/3, I-40129 Bologna BO, Italy \\ ${ }^{3}$ INFN - Istituto Nazionale di Fisica Nucleare, Sezione di Bologna, Viale Berti Pichat 6/2, I-40127 Bologna BO, Italy \\ ${ }^{4}$ SISSA, Via Bonomea 265, I-34136 Trieste, Italy \\ ${ }^{5}$ INFN - Istituto Nazionale di Fisica Nucleare, Sezione di Trieste, Via Bonomea 265, I-34136 Trieste, Italy \\ ${ }^{6}$ Department of Astronomy, University of Washington, 3910 15th Ave NE, WA 98195-1580, Seattle, USA \\ ${ }^{7}$ INAF - Osservatorio Astronomico di Trieste, via Tiepolo 11, I-34143 Trieste, Italy
}

Accepted 2018 October 23. Received 2018 October 23; in original form 2018 September 22

\begin{abstract}
Fuzzy Dark Matter (FDM) represents an alternative and intriguing description of the standard Cold Dark Matter fluid, which is able to explain the lack of direct detection of dark matter particles in the $\mathrm{GeV}$ sector and to alleviate small-scale tensions in the cosmic large-scale structure formation. Cosmological simulations of FDM models in the literature were performed either with very expensive high-resolution grid-based simulations of individual haloes or through $\mathrm{N}$-body simulations encompassing larger cosmic volumes but resorting on significant approximations in the FDM non-linear dynamics to reduce their computational cost. With the use of the new $N$-body cosmological hydrodynamical code AX-GADGET, we are now able not only to overcome such numerical problems, but also to combine a fully consistent treatment of FDM dynamics with the presence of gas particles and baryonic physical processes, in order to quantify the FDM impact on specific astrophysical observables. In particular, in this paper we perform and analyse several hydrodynamical simulations in order to constrain the FDM mass by quantifying the impact of FDM on Lyman $\alpha$ forest observations, as obtained for the first time in the literature in a $N$-body set-up without approximating the FDM dynamics. We also study the statistical properties of haloes, exploiting the large available sample, to extract information on how FDM affects the abundance, the shape, and the density profiles of dark matter haloes.
\end{abstract}

Key words: methods: numerical-dark matter-large-scale structure of Universe.

\section{INTRODUCTION}

In the final decades of the last century, the scientific community consensus gathered around two crucial facts about our Universe, which are now considered the pillars of modern cosmology: first, that the Universe is expanding and it is doing so at an accelerated rate and, secondly, that the estimated baryonic matter content within it cannot account for all the dynamical matter needed to explain its gravitational behaviour.

The standard cosmological framework built upon these concepts, called $\Lambda \mathrm{CDM}$, still holds today. It implies the existence of dark energy, as a source of energy for the accelerated expansion of the Universe, and of dark matter, as an additional gravitational source alongside standard matter, without however specifying their fundamental nature that still represents a major puzzle for cosmologists.

The evidence for a cold and dark form of matter (CDM) - a not-strongly electromagnetically interacting particle or a gravitational quid that mirrors its effect - spans over different scales and is not only related to dynamical properties of systems, as e.g. the inner dynamics of galaxy clusters (Zwicky 1937; Clowe et al. 2006) and the rotation curves of spiral galaxies (Rubin, Ford \& Thonnard 1980; Bosma 1981; Persic, Salucci \& Stel 1996), but also to the gravitational impact on the underlying geometry of spacetime, as strong gravitational lensing of individual massive objects (Koopmans \& Treu 2003) as well as the weak gravitational lensing arising from the large-scale matter distribution (Mateo 1998; Heymans et al. 2013; Planck Collaboration XV 2015; Hildebrandt et al. 2017). Further evidence is based on the relative abundance of matter with respect to the total cosmic energy budget required 
in order to reconcile large-scale structures (LSSs) - as observed through low-redshift surveys - with the angular power spectrum of CMB temperature anisotropies that seed the early-universe density perturbations (as observed, e.g. from WMAP and Planck Komatsu et al. 2011; Planck Collaboration XIII 2016, respectively), on the clustering of luminous galaxies (see e.g. Bel et al. 2014; Alam et al. 2017), on the abundance of massive clusters (Kashlinsky 1998), and their large-scale velocity field (Bahcall \& Fan 1998).

Whether dark matter consists indeed of a yet undetected fundamental particle or it represents an indirect effect of some modification of Einstein's General Relativity theory of gravity is still widely debated. Nevertheless, it has been possible to exclude some of the proposed dark matter effective models, such as e.g. the Modified Newtonian Dynamics and its variants (see e.g. Milgrom 1983; Sanders \& McGaugh 2002; Bekenstein 2004), recently ruled out (Chesler \& Loeb 2017) by the implications of the gravitational wave event GW170817 (Abbott et al. 2017). The lack of detection of dark matter particles in the $\mathrm{GeV}$ mass range through neither indirect astronomical observations (see e.g. Albert et al. 2017), direct laboratory detections (see e.g. Danninger 2017), nor artificial production in high-energy collisions experiments (see e.g. Buonaura 2018) has been undermining the appeal of the most massive dark matter particle candidates, as e.g. the Weakly Interactive Massive Particles, and it is presently shifting the scientific community efforts in the hunt of direct observations from such high-mass ranges towards lower ones (see e.g. Bertone, Hooper \& Silk 2005).

A good starting point where to focus research and to clarify such long-standing uncertainties would be the apparent failures of the $\Lambda \mathrm{CDM}$ model at scales $\lesssim 10 \mathrm{kpc}-$ as given e.g. by the cuspcore problem (Oh et al. 2011), the missing satellite problem (Klypin et al. 1999), the too-big-to-fail problem (Boylan-Kolchin, Bullock \& Kaplinghat 2012) - all arising as an apparent inconsistency between simulations and observations, the latter being more in line with less pronounced density fluctuations at those scales than predicted by the former. However, the nature of such apparent failures has been subject of debate in the astrophysics community. It is still unclear, in fact, whether they should be ascribed to an imperfect baryonic physics implementation in numerical simulations (see e.g. Maccio et al. 2012; Brooks et al. 2013), to an intrinsic diversity of properties related to the formation history and local environment of each individual dark matter halo (Oman et al. 2015), to the fundamental nature of the dark matter particle (see e.g. Kaplinghat, Knox \& Turner 2000; Spergel \& Steinhardt 2000; Rocha et al. 2013; Medvedev 2014), or even to a combination of all these possible causes.

Among the particle candidates that have been proposed in the literature, Fuzzy Dark Matter (FDM) models describe dark matter as made up of very light bosonic particles (see e.g. Hui et al. 2017, for a review on the topic), so light that their quantum nature becomes relevant also at cosmological scales. This requires a description of dark matter dynamics in terms of the Schrödinger equation, in order to take into account quantum corrections, and can be mapped in a fluid-like description where a quantum potential (QP) enters the classical Navier-Stokes equation (Hu, Barkana \& Gruzinov 2000).

The typical wave-like quantum behaviour adds to the standard CDM dynamics a repulsive effective interaction that, along with creating oscillating interference patterns, actively smooths matter overdensities below a redshift-dependent scale that decreases with the cosmic evolution - as confirmed by FDM linear simulations (see e.g. Marsh \& Ferreira 2010; Hlozek et al. 2015) - thus potentially easing some of the previously mentioned small-scale inconsistencies of the CDM model.
The lack of density perturbations at small scales induced by the QP is represented, in Fourier space, by a sharp suppression of the matter power spectrum, which persists - at any given scale - until the action range of the $\mathrm{QP}$ shrinks below such scale and cannot balance any longer the effect of the gravitational potential (see e.g. Marsh 2016b, for another detailed review on the subject). As a matter of fact, while linear theory predicts that perturbations at scales smaller than the cut-off scale never catch up with those at larger scales - untouched by FDM peculiar dynamics - non-linear cosmological simulations have shown that gravity is indeed able to restore intermediate scales to the unsuppressed level, in a sort of healing process (Marsh 2016a; Nori \& Baldi 2018).

FDM non-linear cosmological simulations have been performed over the years either with highly numerically intensive highresolution Adaptive Mesh Refinement (AMR) algorithms able to solve the Schrödinger-Poisson equations over a grid (see e.g. Schive, Tsai \& Chiueh 2010; Schive et al. 2018) or with standard $N$-body codes that, however, include the (linear) suppression only in the initial conditions but neglect the integrated effect of the FDM interaction during the subsequent dynamical evolution (see e.g. Schive et al. 2016; Armengaud et al. 2017; Iršič et al. 2017a) - basically treating FDM as standard dark matter with a suppressed primordial power spectrum, similarly to what is routinely done in Warm Dark Matter simulations (Bode, Ostriker \& Turok 2001). The former approach led to impressive results in terms of resolution (see e.g. Woo \& Chiueh 2009; Schive, Chiueh \& Broadhurst 2014; Veltmaat, Niemeyer \& Schwabe 2018) but is extremely computationally demanding, thereby hindering the possibility of adding a full hydrodynamical description of gas and star formation for cosmologically representative simulation domains. On the other hand, the latter allows for such a possibility because of its reduced computational cost that is, however, gained at the price of the substantial approximation of neglecting QP effects during the simulation (see e.g. Schive et al. 2016).

For these reasons, following the approach first proposed in Mocz \& Succi (2015), we devised AX-GADGET (Nori \& Baldi 2018), a modified version of the $N$-body hydrodynamical cosmological code P-GADGET3 (Springel 2005), to include the dynamical effect of QP through smoothed particle hydrodynamics (SPH) numerical methods. The explicit approximation of the dependence on neighbouring particles results in a less numerically demanding code with respect to full-wave AMR solvers, without compromising cosmological results, with the additional ability to exploit the gas and star physics already implemented in P-GADGET3, along with its more advanced and exotic beyond- $\Lambda \mathrm{CDM}$ extensions such as Modified Gravity (Puchwein, Baldi \& Springel 2013) or Coupled Dark Energy models (Baldi et al. 2010).

Given that gravity, as mentioned above, can restore the suppressed power at intermediate scales in the non-linear regime, major observables related to the LSS at such scales may appear similar in both FDM and CDM picture cosmologies at sufficiently low redshifts. For this reason, Lyman $\alpha$ forest observations could play a crucial role in distinguishing such radically different models of dark matter, being one of the most far reaching direct astrophysical probes in terms of redshift of the LSS observables, sampling the redshift range $z \sim 2-5$ (see e.g. Iršič et al. 2017a, for Lyman $\alpha$ forest analysis in $N$-body simulations, with neglected QP dynamical effects).

In this paper, we performed several simulations with the main goal of studying the effects of FDM on Lyman $\alpha$ forest observations in a fully consistent FDM set-up - i.e. without neglecting the QP during cosmic evolution - in order to constrain the FDM mass. As a by-product of our simulations, we are also able to perform 
an extended analysis of the statistical and structural properties of haloes, exploiting the large statistical sample at our disposal, to extract valuable information about how FDM affects, among others, the halo mass function as well as the shape and density distribution of dark matter haloes.

The paper is organized as follows. In Section 2 we briefly describe the FDM models under consideration, providing all the basic equations that enter our numerical implementation (2.1), and review the theoretical background behind Lyman $\alpha$ forest observations and its physical implications (2.2). In Section 3, we then recall how FDM dynamics is implemented in the AX-GADGET code (3.1); we present the simulation sets performed (3.2) and the strategy used to extract Lyman $\alpha$ information (3.3); in Sections 3.4 and 3.5 the procedures to deal with numerical fragmentation and to match haloes across different simulations are outlined. The results are collected in Section 4 and presented in decreasing order of interested scale, including the matter power spectrum (4.1), the Lyman $\alpha$ statistics (3.3), and the structure characterization (4.3). Finally, in Section 5 we draw our conclusions.

\section{THEORY}

In this section we recall the main properties of a light bosonic field in a cosmological framework, how it affects the growth of LSS, and how Lyman $\alpha$ forest analysis can be used to probe these modifications.

\subsection{FDM models}

The idea of describing dark matter and its key role in the LSS formation in terms of an ultralight scalar particle -i.e. a particle with a mass $\sim 10^{-22} \mathrm{eV} / \mathrm{c}^{2}$ was introduced in $\mathrm{Hu}$ et al. (2000), in which the term FDM was used for the first time and the cosmological implications induced by the quantum behaviour of such a light dark matter field on linear cosmological perturbations were outlined.

The Schrödinger equation describing the dynamics of the bosonic field $\hat{\phi}_{i}$ associated with a single particle can be written as

$i \hbar \partial_{t} \hat{\phi}_{i}=-\frac{\hbar^{2}}{m_{\chi}^{2}} \nabla^{2} \hat{\phi}_{i}+m_{\chi} \Phi \hat{\phi}_{i}$,

where $m_{\chi}$ is the typical mass associated with FDM particles often represented in terms of $m_{22}=m_{\chi} 10^{22} c^{2} / e V-$ and $\Phi$ is the gravitational potential, satisfying the usual Poisson equation

$\nabla^{2} \Phi=4 \pi G a^{2} \rho_{\mathrm{b}} \delta$

with $\delta=\left(\rho-\rho_{\mathrm{b}}\right) / \rho_{\mathrm{b}}$ being the density contrast with respect to the background field density $\rho_{\mathrm{b}}$ (Peebles 1980).

Under the assumption that all the particles belong to a BoseEinstein condensate, the many-body field $\hat{\phi}$ of a collection of particles factorizes and the collective dynamics follows exactly equation (1). If this is the case, it is possible then to express the manybody field $\hat{\phi}$ in terms of collective fluid quantities as density $\rho$ and velocity $\mathbf{v}$, using the Madelung formulation (Madelung 1927)

$\mathbf{v}=\frac{\hbar}{m_{\chi}} \Im \frac{\nabla \hat{\phi}}{\hat{\phi}}$

$\rho=m_{\chi}|\hat{\phi}|^{2}$,

which translates into the usual continuity equation and a modified quantum Navier-Stokes equation reading

$\dot{\mathbf{v}}+(\mathbf{v} \cdot \nabla) \mathbf{v}=-\nabla \Phi+\nabla Q$, where $Q$ is the so-called $\mathrm{QP}$

$Q=\frac{\hbar^{2}}{2 m_{\chi}^{2}} \frac{\nabla^{2} \sqrt{\rho}}{\sqrt{\rho}}=\frac{\hbar^{2}}{2 m_{\chi}^{2}}\left(\frac{\nabla^{2} \rho}{2 \rho}-\frac{|\nabla \rho|^{2}}{4 \rho^{2}}\right)$

also known as quantum pressure if expressed in the equivalent tensorial form as

$\nabla Q=\frac{1}{\rho} \nabla P_{Q}=\frac{\hbar^{2}}{2 m_{\chi}^{2}} \frac{1}{\rho} \nabla\left(\frac{\rho}{4} \nabla \otimes \nabla \ln \rho\right)$.

The additional QP term accounts for the quantum behaviour of particles with a repulsive net effect that counteracts gravitational collapse below a certain scale, related to the Compton wavelength $\lambda_{\mathrm{C}}=\hbar / m_{\chi} c$ identified by the boson mass (Hu et al. 2000). This can be heuristically viewed as the result coming from two combined effects of quantum wave-like nature: decoherence, originating from the Heisenberg uncertainty principle, stirring towards space-filling configurations, and interference-creating oscillatory patterns (Hui et al. 2017).

In an expanding universe described by a scale factor $a$ and the derived Hubble parameter $H=\dot{a} / a$, the linear density perturbation $\delta_{k}$ in Fourier space satisfies - in the comoving frame - the relation

$\ddot{\delta}_{k}+2 H \dot{\delta}_{k}+\left(\frac{\hbar^{2} k^{4}}{4 m_{\chi}^{2} a^{4}}-\frac{4 \pi G \rho_{\mathrm{b}}}{a^{3}}\right) \delta_{k}=0$

that directly sets the typical scale

$k_{Q}(a)=\left(\frac{16 \pi G \rho_{\mathrm{b}} a^{3} m_{\chi}^{2}}{\hbar^{2}}\right)^{1 / 4} a^{1 / 4}$,

where the gravitational pull is balanced by the QP repulsion, sometimes referred as quantum Jeans scale in analogy with the homonym classical one (Chavanis 2012).

The growing solution of equation (8), expressed in terms of the dimensionless variable $x(k, a)=\sqrt{6} k^{2} / k_{Q}^{2}(a)$, is

$D_{+}(x)=\left[\left(3-x^{2}\right) \cos x+3 x \sin x\right] / x^{2}$

whose time dependence is bounded from above and below by the large- and small-scale limits, respectively, as

$D_{+} \propto \begin{cases}a & \text { for } k \ll k_{Q}(a) \\ 1 & \text { for } k \gg k_{Q}(a)\end{cases}$

thereby recovering the standard $\Lambda \mathrm{CDM}$ perturbations evolution at large scales and halting growth of small-scale overdensities (Marsh 2016b).

Structures are unable to collapse until the quantum Jeans scale $k_{Q}(a)$ becomes so little that gravity can overcome the QP repulsive action and, in the linear perturbation regime, will forever carry information about their past suppressed state (Marsh 2016b). In nonlinear simulations, instead, a recovery induced by gravity of the intermediate scales is indeed observed: in terms of matter power spectrum, this implies that a portion of an FDM universe, observed at a fixed scale, will eventually look like a CDM universe if sufficient time for gravity recovery has passed after the crossing of the quantum Jeans scale (as argued also in e.g. Marsh 2016a).

All this considered, it is clear the reason why FDM models peculiar imprints on LSS are to be looked for at very small scales for low redshifts, while larger scales may provide relevant information only as long as higher redshifts are available to observations. In particular, FDM may reveal its presence in the inner part of small collapsed haloes in the form of a flat solitonic core (see e.g. Schive et al. 2014; Marsh \& Pop 2015; De Martino et al. 2018; Lin et al. 
2018) while larger scales may show FDM imprints in the highredshift gas distribution (Armengaud et al. 2017; Kobayashi et al. 2017; Iršič et al. 2017a).

\subsection{Lyman $\alpha$ forest}

The Lyman $\alpha$ forest is the main manifestation of the intergalactic medium (IGM), the diffuse filamentary matter filling the space between galaxies, and it constitutes a very powerful method for constraining the properties of DM in the small-scale $\left(0.5 \mathrm{Mpc} h^{-1} \lesssim\right.$ $\left.\lambda \lesssim 20 \mathrm{Mpc} h^{-1}\right)$ and high-redshift regime $(2 \lesssim z \lesssim 5$ ) (see e.g. Viel et al. 2005, 2013). The physical observable for Lyman $\alpha$ experiments is the flux power spectrum $P_{\mathrm{F}}(k, z)$. Constraints on the matter power spectrum from Lyman $\alpha$ forest data at small cosmological scales are only limited by the thermal cut-off in the flux power spectrum, introduced by pressure and thermal motions of baryons in the photoionized IGM. That is why this astrophysical observable has provided some of the tightest constraints to date on DM scenarios featuring a small-scale power suppression (Iršič et al. 2017c; Murgia, Iršič \& Viel 2018), including FDM models, both in the case where they constitute the entire DM (Armengaud et al. 2017; Iršič et al. 2017a) and in the case in which they are a fraction of the total DM amount (Kobayashi et al. 2017).

Ultralight scalar DM candidates are indeed expected to behave differently with respect to standard CDM on scales of the order of their de Broglie wavelength, where they induce a suppression of the structure formation, due to their wave-like nature. In particular, for FDM particles with masses $\sim 10^{-22} \mathrm{eV}$, such a suppression occurs on (sub)galactic scales, being thereby the ideal target for Lyman $\alpha$ forest observations. Moreover, as we discussed in the previous section, Lyman $\alpha$ forest observations probe a redshift and scales range in which the difference between $\Lambda \mathrm{CDM}$ and the FDM models - for the masses considered - is highly significant.

All the limits found in the literature on FDM parameters - i.e. the mass $m_{\chi}-$ using Lyman $\alpha$ observations (as e.g. Armengaud et al. 2017; Kobayashi et al. 2017; Iršič et al. 2017a) have been computed by assuming that ultralight scalars behave as standard pressureless CDM and by comparing Lyman $\alpha$ data with flux power spectra obtained from standard SPH cosmological simulations, which completely neglected the QP effects during the non-linear structure evolution. In other words, the non-standard nature of the dark matter candidate was simply encoded in the suppressed initial conditions used as inputs for performing the hydrodynamical simulations.

One of the goals of this work is to use AX-GADGET in order to provide the first fully accurate constraints on the FDM mass, by going beyond the standard dynamical approximation of ignoring the time-integrated QP effect. Including such an effect in our numerical simulations is thereby expected to tighten the limits published so far in the literature, since it introduces a repulsive effect at small scales throughout the simulation evolution that contributes to the matter power spectrum suppression. Besides presenting the new constraints, we will also carry out a meticulous comparison with the bounds determined under the aforementioned approximation, in order to exactly quantify its validity.

\section{NUMERICAL METHODS}

In this section we briefly review the implementation of the AXGADGET code routines that are devoted to the FDM dynamics (an in-depth description featuring analytic and cosmological tests can be found in Nori \& Baldi 2018). We then continue presenting how Lyman $\alpha$ forest observations are modelled and extracted from numerical simulations. Finally, we describe our approach to discriminate spurious haloes - which are expected to form in particlebased simulations featuring a suppressed power spectrum (see e.g. Wang \& White 2007), such as Warm Dark Matter, Hot Dark Matter, or FDM models - from genuine ones, in order to properly take into account the known problem of numerical fragmentation, together with the strategy we used to cross-match haloes in the different simulations.

\subsection{The code: AX-GADGET}

AX-GADGET is a module available within the cosmological and hydrodynamical $N$-body code P-GADGET3, a non-public extension of the public GADGET2 code (Springel 2005). It features a new type of particle in the system - i.e. ultralight axion - whose strongly non-linear quantum dynamics is solved through advanced and refined SPH routines, used to reconstruct the density field from the particle distribution and, therefore, to calculate the QP contribution to particle acceleration.

The general SPH approach relies on the concept that the density field $\rho$ underlying a discrete set of particles can be approximated at particle $i$ position with the weighted sum of the mass $m$ of neighbouring particles $\mathrm{NN}(i)$

$\rho_{i}=\sum_{j \in \mathrm{NN}(i)} m_{j} W_{i j}$

where the mass is convolved with a kernel function $W_{i j}$ of choice, characterized by a particle-specific smoothing length $h_{i}$, and whose extent is fixed imposing

$\frac{4}{3} \pi h_{i}^{3} \rho_{i}=\sum_{j \in \mathrm{NN}(i)} m_{j}$

so that only a given mass is enclosed within it.

Once the density field is reconstructed, every observable is locally computed through weighted sums as

$O_{i}=\sum_{j \in \mathrm{NN}(i)} m_{j} \frac{O_{j}}{\rho_{j}} W_{i j}$

and its derivatives are iteratively obtained with

$\nabla O_{i}=\sum_{j \in \mathrm{NN}(i)} m_{j} \frac{O_{j}}{\rho_{j}} \nabla W_{i j}$,

where the derivative is applied on the window function.

The exact scheme of the SPH algorithm is not fixed, since each observable can be expressed in many analytically equivalent forms that, however, translate into different operative summations. For example, the QP of equation (6) can be calculated using recursive derivatives of $\rho, \sqrt{\rho}$ or $\log \rho$ intermediate observables. An important consequence of such flexibility is that different but analytically equivalent expressions will map into operative sums that carry different numerical errors. Among the several strategies that have been employed in the literature to reduce the residual numerical errors (see e.g. Brookshaw 1985; Cleary \& Monaghan 1999; Colin, Egli \& Lin 2006), the following has proven the more stable and accurate for the QP case (see Nori \& Baldi 2018, for a comparison between different implementations), and will therefore be the one of our choice:

$\nabla \rho_{i}=\sum_{j \in \mathrm{NN}(i)} m_{j} \nabla W_{i j} \frac{\rho_{j}-\rho_{i}}{\sqrt{\rho_{i} \rho_{j}}}$ 


$$
\begin{aligned}
\nabla^{2} \rho_{i} & =\sum_{j \in \mathrm{NN}(i)} m_{j} \nabla^{2} W_{i j} \frac{\rho_{j}-\rho_{i}}{\sqrt{\rho_{i} \rho_{j}}}-\frac{\left|\nabla \rho_{i}\right|^{2}}{\rho_{i}} \\
\nabla Q_{i} & =\frac{\hbar^{2}}{2 m_{\chi}^{2}} \sum_{j \in \mathrm{NN}(i)} \frac{m_{j}}{f_{j} \rho_{j}} \nabla W_{i j}\left(\frac{\nabla^{2} \rho_{j}}{2 \rho_{j}}-\frac{\left|\nabla \rho_{j}\right|^{2}}{4 \rho_{j}^{2}}\right) .
\end{aligned}
$$

AX-GADGET has undergone various stability tests and has proven to be not only less numerically intensive with respect to AMR full-wave solvers (Schive et al. 2010), due to the intrinsic SPH local approximation, but also to be accurate for cosmologically relevant scales as it agrees both with the linear (Hlozek et al. 2015) and non-linear results (Woo \& Chiueh 2009) available in the literature, even if a proper convergence and code comparison test has not yet been performed, since it would be necessary to assess the consistency of different numerical methods at very small scales.

In fact, while cosmological and analytical results, as e.g. the soliton formation, are well recovered by $N$-body simulations, interference patterns emerging at very small scales seem more challenging to be represented accurately, due to their oscillatory nature that can be overly smoothed if the resolution, i.e. the number of particles, used is too low. $N$-body simulations at very high resolution, i.e. to the pc level, have yet to be performed, but as also argued in more detail in Appendix A, it is our opinion that whether interference patterns can be observed or not is ultimately a matter of resolution.

The implementation of FDM physics in AX-GADGET includes the possibility to simulate universes with multiple CDM and FDM species or FDM particles with self- or external interactions, as recently included with the merging of the AX-GADGET module with the C-Gadget module of Coupled Dark Matter models (Baldi et al. 2010).

Moreover, AX-GADGET inherits automatically all the large collection of physical implementations ranging from gas cooling and star formation routines to Dark Energy and Modified Gravity implementations - which have been developed for P-GADGET3 by a wide range of code developers.

All these properties allow us to investigate a yet unexplored wide variety of extended FDM models and make of AX-GADGET a valuable tool complementary to high-resolution AMR codes - to study the effects of FDM on LSS formation and evolution. In this work, we consider the simplest non-interacting case with the totality of the dark matter fluid composed by FDM.

\subsection{Simulations}

In this work, we performed two sets of simulations, for a total number of 14 cosmological runs. The first set consists in DM-only simulations used to characterize the small-scale structures at low redshift, i.e. down to $z=0-$ while the second one is evolved to $z=$ 2 and includes gas particles and a simplified hydrodynamical treatment, as described in Section 3.1, specifically developed for Lyman $\alpha$ forest analyses (the so-called 'QLYA', or Quick-Lyman-alpha method, see Viel, Haehnelt \& Springel 2004). Both sets consist in three pairs of simulations, one pair for each considered FDM mass, evolved either including or neglecting the effect of the QP in the dynamics labelling these two cases as FDM and FDMnoQP, respectively - in order to assess and quantify the entity of such an approximation often employed in the literature.

Both sets of simulations follow the evolution of $512^{3}$ dark matter particles in a comoving periodic box with a side length of $15 \mathrm{Mpc}$, using $1 \mathrm{Kpc}$ as gravitational softening. The mass resolution for the dark-matter-only simulations is $2.2124 \times 10^{6} \mathrm{M}_{\odot}$. In all cases we generate initial conditions at $z=99$ using the 2LPTIC code (Crocce, Pueblas \& Scoccimarro 2006), which provides initial conditions for cosmological simulations by displacing particles from a cubic Cartesian grid following a second-order Lagrangian Perturbation Theory-based approach, according to a random realization of the suppressed linear power spectrum as calculated by AXIONCAMB (Hlozek et al. 2015) for the different FDM masses under investigation. To ensure a coherent comparison between simulations, we used the same random phases to set up the initial conditions. In particular, the FDM masses $m_{\chi}$ considered here are $2.5 \times 10^{-22}$, $5 \times 10^{-22}$, and $2.5 \times 10^{-21} \mathrm{eV} / \mathrm{c}^{2}$, in order to sample the mass range preferred by the first Lyman $\alpha$ constraints in the literature (see in particular Armengaud et al. 2017; Kobayashi et al. 2017; Iršič et al. 2017a), obtained through $N$-body simulations with approximated dynamics.

Cosmological parameter used are $\Omega_{\mathrm{m}}=0.317, \Omega_{\Lambda}=0.683, \Omega_{\mathrm{b}}=$ 0.0492 , and $H_{0}=67.27 \mathrm{~km} \mathrm{~s}^{-1} \mathrm{Mpc}^{-1}, A_{\mathrm{s}}=2.20652 \times 10^{-9}$, and $n_{\mathrm{s}}=0.9645$. A summary of the simulation specifications can be found in Table 1.

\subsection{Lyman $\alpha$ forest}

The flux power spectrum $P_{F}(k, z)$ is affected both by astrophysical and cosmological parameters. It is therefore crucial to accurately quantify their impact in any investigation involving the flux power as a cosmological observable. To this end, our analysis is based on a set of full hydrodynamical simulations that provide a reliable template of mock flux power spectra to be compared with observations.

For the variations of the mean Lyman $\alpha$ forest flux, $\bar{F}(z)$, we have explored models up to 20 per cent different than the mean evolution given by Viel et al. (2013).

We have varied the thermal history of the IGM in the form of the amplitude $T_{0}$ and the slope $\gamma$ of its temperature-density relation, generally parametrized as $T=T_{0}(1+\delta)^{\gamma-1}$, with $\delta$ being the IGM overdensity (Hui \& Gnedin 1997). We have then considered a set of three different temperatures at mean density, $T_{0}(z=4.2)=$ 7200, $11000,14800 \mathrm{~K}$, which evolve with redshift, as well as a set of three values for the slope of the temperature-density relation, $\gamma(z=4.2)=1.0,1.3$, and 1.5. The reference thermal history has been chosen to be defined by $T_{0}(z=4.2)=11000$ and $\gamma(z=$ $4.2)=1.5$, providing a good fit to observations (Bolton et al. 2017). Following the conservative approach of Iršič et al. (2017a), we have modelled the redshift evolution of $\gamma$ as a power law, such that $\gamma(z)=\gamma^{A}\left[(1+z) /\left(1+z_{\mathrm{p}}\right)\right]^{\gamma^{S}}$, where the pivot redshift $z_{\mathrm{p}}$ is the redshift at which most of the Lyman $\alpha$ forest pixels are coming from (i.e. $z_{\mathrm{p}}=4.2$ for MIKE/HIRES + XQ-100). However, in order to be agnostic about the thermal history evolution, we let the amplitude $T_{0}(z)$ free to vary in each redshift bin, only forbidding differences greater than $5000 \mathrm{~K}$ between adjacent bins (Iršič et al. 2017c).

Furthermore, we have also explored several values for the cosmological parameters $\sigma_{8}$, i.e. the normalization of the matter power spectrum, and $n_{\text {eff }}$, namely the slope of the matter power spectrum at the scale of Lyman $\alpha$ forest $\left(0.009 \mathrm{~s} \mathrm{~km}^{-1}\right)$, in order to account for the effect on the matter power spectrum due to changes in its initial slope and amplitude (McDonald et al. 2006; Seljak, Slosar \& McDonald 2006; Arinyo-i Prats et al. 2015). We have therefore considered five different values for $\sigma_{8}$ (in the interval $[0.754,0.904]$ ) and $n_{\text {eff }}$ (in the range $[-2.3474,-2.2674]$ ). 
Table 1. Summary of the properties of the simulations set used for structure characterization.

\begin{tabular}{lccccc}
\hline Model & QP in dynamics & $m_{\chi}\left(10^{-22} \mathrm{eV/c} \mathrm{c}^{2}\right)$ & $N$ haloes & $N$ genuine haloes & $M_{\text {cut }}\left(10^{10} \mathrm{M}_{\odot}\right)$ \\
\hline LCDM & $\times$ & - & 57666 & 56842 & - \\
FDM-25 & $\checkmark$ & 25 & 25051 & 13387 & 0.04056 \\
FDM-5 & $\checkmark$ & 5 & 10058 & 2736 & 0.1645 \\
FDM-2.5 & $\checkmark$ & 2.5 & 8504 & 1301 & 0.3151 \\
FDMnoQP-25 & $\times$ & 25 & 25432 & 13571 & 0.04056 \\
FDMnoQP-5 & $\times$ & 5 & 10376 & 2856 & 0.1645 \\
FDMnoQP-2.5 & $\times$ & 2.5 & 8819 & 1374 & 0.3151 \\
\hline
\end{tabular}

We have also varied the re-ionization redshift $z_{\text {rei }}$, for which we have considered the three different values $z_{\text {rei }}=7,9$, and 15 , with $z_{\text {rei }}=9$ being the reference value and, finally, we have considered ultraviolet (UV) fluctuations of the ionizing background, which may have non-negligible effects at high redshift. The amplitude of this phenomenon is parametrized by the parameter $f_{\mathrm{UV}}$ : the corresponding template is built from a set of three models with $f_{\mathrm{UV}}=0,0.5$, and 1 , where $f_{\mathrm{UV}}=0$ is associated with a spatially uniform UV background.

Based on the aforementioned grid of simulations, we have performed a linear interpolation between the grid points in such multidimensional parameter space, to obtain predictions of flux power for the desired models.

We have to note that the thermal history implementation of Iršič et al. (2017a) and the one used in this work are slightly different. For this reason, since the simulations of the grid were performed without the introduction of the QP in the dynamics, we mapped our results into the grid ones using the ratio between FDM and FDMnoQP simulations. This is, of course, not an exact procedure but we assume that the ratio of flux power spectrum with and without quantum pressure is relatively insensitive to the thermal history (Murgia et al. 2018).

In order to constrain the various parameters, we have used a data set given by the combination of intermediate- and high-resolution Lyman $\alpha$ forest data from the XQ-100 and the HIRES/MIKE samples of QSO spectra, respectively. The XQ-100 data are constituted by a sample of medium resolution and intermediate-signal-to-noise QSO spectra, obtained by the XQ-100 survey, with emission redshifts $3.5 \leq z \leq 4.5$ (López S. et al. 2016). The spectral resolution of the X-shooter spectrograph is $30-50 \mathrm{~km} \mathrm{~s}^{-1}$, depending on the wavelength. The flux power spectrum $P_{\mathrm{F}}(k, z)$ has been calculated for a total of $133(k, z)$ data points in the ranges $z=3$, $3.2,3.4,3.6,3.8,4,4.2$, and 19 bins in $k$-space in the range 0.003 $0.057 \mathrm{~s} \mathrm{~km}^{-1}$ (see Iršič et al. 2017b, for a more detailed description). MIKE/HIRES data are instead obtained with the HIRES/KECK and the MIKE/Magellan spectrographs, at redshift bins $z=4.2,4.6,5.0$, 5.4 and in $10 k$-bins in the interval $0.001-0.08 \mathrm{~s} \mathrm{~km}^{-1}$, with a spectral resolution of 13.6 and $6.7 \mathrm{~km} \mathrm{~s}^{-1}$, for HIRES and MIKE, respectively (Viel et al. 2013). As in the analyses by Viel et al. (2013) and Iršič et al. (2017c), we have imposed a conservative cut on the flux power spectra obtained from MIKE/HIRES data, and only the measurements with $k>0.005 \mathrm{~s} \mathrm{~km}^{-1}$ have been used, in order to avoid possible systematic uncertainties on large scales due to continuum fitting. Furthermore, we do not consider the highest redshift bin for MIKE data, for which the error bars on the flux power spectra are very large (see Viel et al. 2013, for more details). We have thus used a total of $182(k, z)$ data points. Parameter constraints are finally obtained with a Monte Carlo Markov Chain (MCMC) sampler that samples the likelihood space until convergence is reached.

\subsection{Numerical fragmentation}

For cosmological models whose LSS properties depart sensibly from $\Lambda$ CDM only at small scales as FDM models, the thorough analysis of the statistical overall properties and the specific inner structures of haloes represents the most relevant and often largely unexploited source of information. In $\mathrm{N}$-body simulations, this implies the use of a suitable clustering algorithm to build a halo catalogue in order to identify gravitationally bound structures that can then be studied in their inner structural properties.

In this work, we rely on the SUBFIND routine already implemented in P-GADGET3, a two-step halo finder that combines a friends-offriends (FoF) algorithm (Davis et al. 1985) to find particle clusters that defines the primary structures of our halo sample - with an unbinding procedure to identify gravitationally bound substructures within the primary haloes (Springel et al. 2001). Hereafter, we use the term primary structures to identify the substructures of each FoF group containing the most gravitationally bound particle, $s u b$ haloes for the non-primary structures and haloes when we generally consider the whole collection of structures found.

However, a long-standing problem that affects $N$-body simulations, when characterized by a sharp and resolved cut-off of the matter power spectrum, has to be taken into account in the process of building a reliable halo sample. This is the so-called numerical fragmentation, i.e. the formation of artificial small-mass spurious clumps within filaments (see e.g. Wang \& White 2007; Schneider et al. 2012; Angulo, Hahn \& Abel 2013; Lovell et al. 2014; Schive et al. 2016).

While it has been initially debated whether the nature of such fragmentation was to be considered physical or numerical, the detailed analysis by Wang \& White (2007) showed that in Warm and Hot Dark Matter simulations (as e.g. Bode et al. 2001), which are characterized by a highly suppressed matter power spectrum, the formation of small-mass subhaloes was resolution dependent and related to the large difference between force resolution and mean particle separation (as already suggested by Melott \& Shandarin 1989).

To identify spurious haloes in simulations and select a clean sample to study and characterize the structures of FDM haloes in each simulation, we take cue from the procedure outlined in Lovell et al. (2014): in particular, we use the mass at low redshift and the spatial distribution of particles as traced back in the initial conditions as proxies for the artificial nature of haloes as described below.

In fact, the more the initial power spectrum is suppressed at small scales, the more neighbouring particles are coherently homogeneously distributed, thus facilitating the onset of artificially bounded and small ensembles that eventually outnumber the physical ones. As already shown by Wang \& White (2007), the dimensionless power spectrum peak scale $k_{\text {peak }}$ and the resolution of the 
simulation, i.e. described through the mean inter-particle distance $d$, can be related together to get the empirical estimate

$M_{\text {lim }}=10.1 \rho_{\mathrm{b}} d / k_{\text {peak }}^{2}$

describing the mass at which most of the haloes have a numerical rather than a physical origin. In Lovell et al. (2014), this mass is used as a pivotal value for the mass $M_{\mathrm{CUT}}$ used to discriminate genuine and spurious haloes lying above and below such a threshold, respectively - which is set as $M_{\mathrm{CUT}}=0.5 M_{\mathrm{lim}}$.

In addition to the mass discriminating criterion, Lovell et al. (2014) showed that particles that generate spurious haloes belong to degenerate regions in the initial conditions and are more likely to lie within filaments, stating that the reconstructed shape of the halo particles ensemble in the initial conditions can be used to identify spurious structures. $N$-body initial conditions are generally designed as regularly distributed particles on a grid from which are displaced in order to match the desired initial power spectrum. Hence, numerical fragmentation originates mostly from particles lying in small planar configurations, belonging to the same row/column domain or a few adjacent ones.

Therefore, we need a method to quantitatively describe the shape of subhaloes and of the distribution of their member particles once traced back to the initial conditions of the simulation. To this end, we resort to the inertia tensor of the particle ensemble

$I_{i j}=\sum_{\text {particles }} m\left(\hat{e}_{i} \cdot \hat{e}_{j}\right)|r|^{2}-\left(\mathbf{r} \cdot \hat{e}_{i}\right)\left(\mathbf{r} \cdot \hat{e}_{j}\right)$,

where $m$ and $\mathbf{r}$ are the particle mass and position, respectively, and $\hat{e}$ are the unit vectors of the reference orthonormal base. The eigenvalues and the eigenvectors of the inertia tensor represent the square moduli and unitary vectors of the three axes of the equivalent triaxial ellipsoid with a uniform mass distribution. We define $a \geq$ $b \geq c$ the moduli of the three axes and the sphericity $s=c / a$ as the ratio between the minor and major ones: a very low sphericity will characterize the typical degenerate domains of numerical fragmentation.

For these reasons, we use the combined information carried by the mass and the sphericity in the initial condition to clean the halo catalogues from spurious ones by applying independent cuts on both quantities as will be detailed below.

In Fig. 1 the mass-sphericity distributions of the different simulations are plotted at $z=0$ (upper left panel) and at $z=99$ (upper right panel) where each point represents a halo identified by SUBFIND, without applying any selection. Solid and dash-dotted lines denote the median and the 99th percentile of the distribution; in the side panels we display the cumulative distributions, where the contribution of spurious haloes is highlighted in black.

By looking at the two panels, it is possible to notice that the total cumulative sphericity distribution at low redshift is fairly model independent, so that distinguishing spurious haloes from genuine ones is impossible. However, if we trace the particle ensembles of each halo found at $z=0$ back to the initial conditions at redshift $z=99$, using particles ID, and we study the resulting reconstructed masssphericity relation, the anomalous component of the distribution associated with spurious haloes clearly emerges as a low-sphericity peak, which is more pronounced for smaller values of the FDM particle mass.

In fact, as the mass $m_{\chi}$ decreases, the smoothing action of the QP becomes more efficient, inducing homogeneity at larger and larger scales in the initial conditions and increasing, consequently, the contamination of numerical fragmentation. It clearly appears that the population of haloes in the initial conditions is homogeneously distributed in $\Lambda \mathrm{CDM}$ while a bimodal structure emerges at lower and lower FDM mass. In particular, an increasing number of haloes are located in a small region characterized by low mass $(M \lesssim$ $\left.10^{9} \mathrm{M}_{\odot}\right)$ and low sphericity $(s \lesssim 0.20)$.

As there is no theoretical reason why the QP should favour the collapse of ensembles with very low sphericities in the initial conditions with respect to the $\Lambda \mathrm{CDM}$ case, we consider this second population as the result of numerical fragmentation.

As in Lovell et al. (2014), we choose to compute $M_{\mathrm{CUT}}=0.5 M_{\mathrm{lim}}$ using equation (19), one $M_{\mathrm{CUT}}$ for each value of the FDM mass, as reported in Table 1, that defines the upper bound of the discarded mass regions, i.e. the black-shaded areas in all the panels of Fig. 1. It is interesting to notice that the masses $M_{\mathrm{CUT}}$ appear to be very close to the values at which the sphericity medians of the simulation sample - in the initial conditions - depart from the ones of $\Lambda \mathrm{CDM}$, as can be seen in the lower right panel of Fig. 1 . As the $M_{\mathrm{CUT}}$ values we obtain are slightly larger compared to these departing values, we confirm the choice of the former over the latter, as a more conservative option for the mass thresholds dividing spurious from genuine haloes.

In Lovell et al. (2014), the selection in terms of initial sphericity was operationally performed discarding every halo with a sphericity lower than $s_{\text {CUT }}=0.16$, equal to the 99th percentile of the distribution of haloes with more than 100 particles in the $\Lambda \mathrm{CDM}$ simulation. In our set of simulations, a similar value denotes the 99th percentile as measured at the $M_{\mathrm{CUT}}$ mass in each simulation, so we adopt it as our own threshold in sphericity. Let us stress that the haloes that are discarded through sphericity selection in the initial conditions have sphericities at $z=0$ that are statistically consistent with the genuine sample, making their numerical origin impossible to notice based only on the sphericity distribution at $z=$ 0 . However, the mass constraint is far more rigid than the sphericity one in all models but $\Lambda \mathrm{CDM}$, where no mass limit is imposed.

Finally, in Table 2 we have summarized the comparison of the number of haloes in the FDMnoQP set-up with respect to the corresponding FDM set-up, presented as the ratio of the total number of haloes found by SUBFIND and the number of genuine haloes remaining after the exclusion of spurious ones. It is possible to see that in the FDMnoQP simulations, for the three FDM masses considered, the total number of haloes is overestimated by a factor of $\sim 2.5$ per cent on average while the genuine haloes excess becomes more important as the FDM mass decreases, up to 5.6 per cent for $m_{22}=2.5$. This means that neglecting the effects of the QP during the simulation leads to the formation of haloes that are not present when the full QP dynamics is taken into account and that, using our à la Lovell et al. (2014) spurious detection selection, such haloes pass the numerical fragmentation test and contaminate any halo statistical property characterization.

\subsection{Inter-simulations halo matching}

In FDM models, as we said in the previous sections, not only the initial power spectrum of matter perturbation is suppressed at small scales, thereby preventing the formation of small-mass structures, but the dynamical evolution of density perturbations changes due to the effect of the QP, intimately affecting the development of structures during the whole cosmological evolution by opposing gravitational collapse. The implementation of such an effect in AX-GADGET breaks the one-to-one correspondence of the spatial position of collapsed structures in simulations with different FDM masses - especially for smaller objects - despite the identical random phases used to set up the initial conditions. 

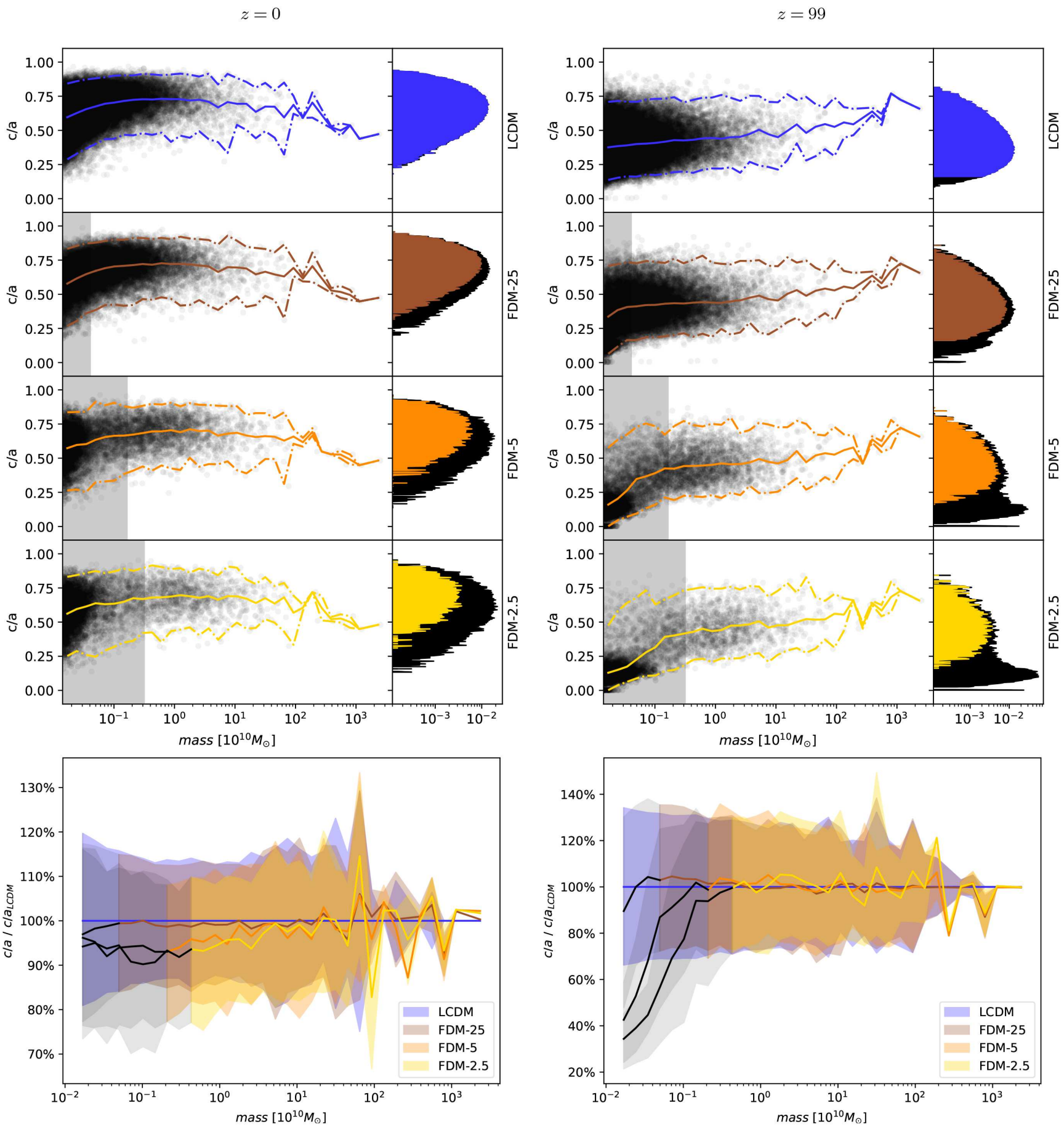

Figure 1. Sphericities of all dark matter particle ensembles as found by SUBFIND as a function of their mass (upper panels) at redshifts $z=0$ and 99 (leftand right-hand panels, respectively). The black-shaded area represents the discarded region below the different mass cuts $M_{\mathrm{CUT}}$, corresponding to each model. Each black dot represents a subhalo and the solid (dot-dashed) lines describe the median (99th percentile) of the total distribution, which are all gathered and contrasted with $\Lambda \mathrm{CDM}$ in the lower panel. The total sphericity distribution - integrated in mass - is represented in the side panels where the contribution of the discarded sample to medians and distributions are portrayed in black. Lower panels feature the median of the mass-sphericity distributions, presented as the ratio with respect to $\Lambda \mathrm{CDM}$. The shaded areas, corresponding to the $\pm 1 \sigma$ of the distribution, are colour coded as in the upper panels. The blackened median and shaded areas represent the excluded portion of the sphericity distributions below the corresponding $M_{\mathrm{CUT}}$.

We indeed expect bigger haloes not to change dramatically their position at low redshift across different simulations, while this is not the case for lighter subhaloes that are more affected by the evolving local non-linear balance between gravity and the QP of the environment.
This makes it more difficult to identify matching collapsed objects of common origin across the simulations, and to study how FDM models affect the inner structure of haloes on a halo-to-halo basis. 
Table 2. The total and genuine number of haloes, presented as the ratio between the simulations neglecting and considering the QP dynamical effects.

\begin{tabular}{lcc}
\hline$m_{\chi}\left(10^{-22} e V / c^{2}\right)$ & $N$ haloes(\%) & $N$ genuine haloes(\%) \\
\hline 25 & 101.6 & 101.4 \\
5 & 103.5 & 104.4 \\
2.5 & 103.1 & 105.6 \\
\hline
\end{tabular}

Table 3. Number of common matches across LCDM and FDM simulations, using different values of the parameter $\tilde{M}$ representing the minimum allowed ratio between the minimum and maximum masses of each candidate couple.

\begin{tabular}{lcc}
\hline$\tilde{M}$ & $m_{\min } / m_{\max }(\%)$ & $N$ matches \\
\hline $1 / 39$ & 95 & 53 \\
$1 / 19$ & 90 & 162 \\
$3 / 37$ & 85 & 234 \\
$1 / 9$ & 80 & 279 \\
$1 / 7$ & 75 & 304 \\
$1 / 3$ & 50 & 346 \\
$3 / 5$ & 25 & 361 \\
1 & 0 & 389 \\
\hline
\end{tabular}

To this end, we devise an iterative matching procedure, to be repeated until no more couples are found, as the following: given a halo $i$ at position $\mathbf{r}_{i}$ and total mass $m_{i}$ in simulation $A$,

(i) select all haloes $j$ belonging to simulation $B$ as potential counterparts if $\left|\mathbf{r}_{i}-\mathbf{r}_{j}\right| /\left(a_{i}+a_{j}\right)<\tilde{R}$, where $a_{i}$ and $a_{j}$ are the major axes of the haloes computed through the inertia tensor of all their member particles.

(ii) within the ensemble selected at the previous point, retain only the haloes $k \subseteq j$ whose masses satisfy the condition $\left|m_{i}-m_{k}\right| /\left(m_{i}+\right.$ $\left.m_{k}\right)<\tilde{M}$.

(iii) if more than one halo $l k$ is left, then choose the one for which $\left|\mathbf{r}_{i}-\mathbf{r}_{l}\right| /\left(a_{i}+a_{l}\right)$ is minimum.

(iv) after having considered all the haloes in $A$, if more than one are linked to the same halo $l$ belonging to $B$, choose the couple $(i, l)$ that minimizes $\left[\left|\mathbf{r}_{i}-\mathbf{r}_{l}\right| /\left(a_{i}+a_{l}\right)\right]^{2}+\left[\left|m_{i}-m_{l}\right| /\left(m_{i}+m_{l}\right)\right]^{2}$, in order give the same weight to the two criteria.

This method is flexible enough to account for the shift in mass and position we expect from simulations with different FDM mass models, but conservative enough to ensure the common origin of the subhalo couples. Moreover, using the combination of position and mass filters, we are able to discriminate couples in all mass ranges: position filtering is a weaker constraint in the case of bigger haloes since they occupy a big portion of a simulation where instead the mass filter is very strict; vice versa, it is more powerful for smaller haloes for which the mass filter selects a large number of candidates.

Operatively, we use the previous procedure to match haloes in each simulation with the $\Lambda \mathrm{CDM}$ one and we refer to the subset of haloes that share the same $\Lambda \mathrm{CDM}$ companion across all the simulations as the common sample.

For geometrical reasons, we set the limit value for $\tilde{R}$ to be 0.5 : this represents the case in which two haloes with the same major axis $a$ have centres separated exactly by the same amount $a$. The configurations that are selected by point $(i)$ are the ones for which the distance between the halo centres is less or equal to the smallest major axis between the two. A higher value for $\tilde{R}$ would include genuine small haloes that have been more subject to dynamical QP drifting but would also result in a spurious match of bigger haloes. For these reasons, we adopt $\tilde{R}=0.5$, checking that the selected sample gains or loses $\sim 5$ per cent of components if values 0.45 and 0.55 are used, without modifying the overall statistical properties of the sample itself.

With respect to $\tilde{M}$ at point (ii), instead, we applied the matching algorithm using several values, each denoting a specific threshold of the minimal value allowed for the mass ratio of halo couples in order to be considered as a match. As reported in Table 3, more than 60 per cent of all the matching haloes across LCDM and FDM simulations without mass selection $\tilde{M}=1$ case have a mass ratio in the $100-85$ per cent ratio range and almost 80 per cent in the 100-75 per cent range. In order not to spoil our matching catalogue, especially with very close but highly different in mass halo couples, we choose the limiting value of $\tilde{M}=1 / 7$.

\section{RESULTS}

In this section we present the results obtained from our simulations in decreasing order of scale involved, starting from the matter power spectrum, to the simulated Lyman $\alpha$ forest observations, to the statistical characterization of halo properties, and their density profiles.

\subsection{Matter power spectrum}

The relative difference of the matter power spectrum of the various FDM models with respect to $\Lambda \mathrm{CDM}$ at four different redshifts is displayed in Fig. 2.

As already found in the literature (see e.g. Marsh 2016a; Nori \& Baldi 2018), the evolution of the matter power spectrum shows that the initial suppression encoded in the transfer functions used to build up the initial conditions is restored at intermediate scales to the unsuppressed level, eventually, by the non-linear gravitational evolution.

At the redshifts and scales that are relevant for Lyman $\alpha$ forest observations, however, the relative suppression with respect to $\Lambda \mathrm{CDM}$ is still important and ranges from 5 to 20 per cent for the lowest FDM mass considered.

The relative difference of the matter power spectrum, displayed in Fig. 3, shows an additional suppression with respect to $\Lambda \mathrm{CDM}$ when the QP is included in the dynamical evolution in the comparison between the FDM and FDMnoQP simulations, e.g. comparing Figs 2 and 3 we find that $(F D M / L C D M-1) /(F D M n o Q P / L C D M-$ $1) \approx 110-115$ per cent for the $m_{22}=2.5$ case at $k \sim 10 \mathrm{~h} \mathrm{Mpc}^{-1}$. This is consistent with the QP full dynamical treatment contributing as an integrated smoothing force that contrasts the gravitational collapse of the otherwise purely collisionless dynamics.

\subsection{Lyman $\alpha$ forest flux statistics}

In order to build our simulated Lyman $\alpha$ observations, we extracted 5000 mock forest spectra from random line of sights within the simulated volume. The spectra are extracted according to SPH interpolation and the ingredients necessary to build up the transmitted flux are the HI-weighted peculiar velocity, temperature, and neutral fraction. Among the different flux statistics that can be considered, we focus on the flux probability distribution function (PDF) and flux power spectrum. Unless otherwise stated, we normalize the extracted flux arrays in order to have the same observed mean flux over the whole sample considered and for all the simulations. In any case, we do find that the scaling factor for the optical depth arrays over the whole simulated volume is $1.6,1.4$, and 1.1 times higher than in the $\Lambda \mathrm{CDM}$ case in order to achieve the same mean flux 

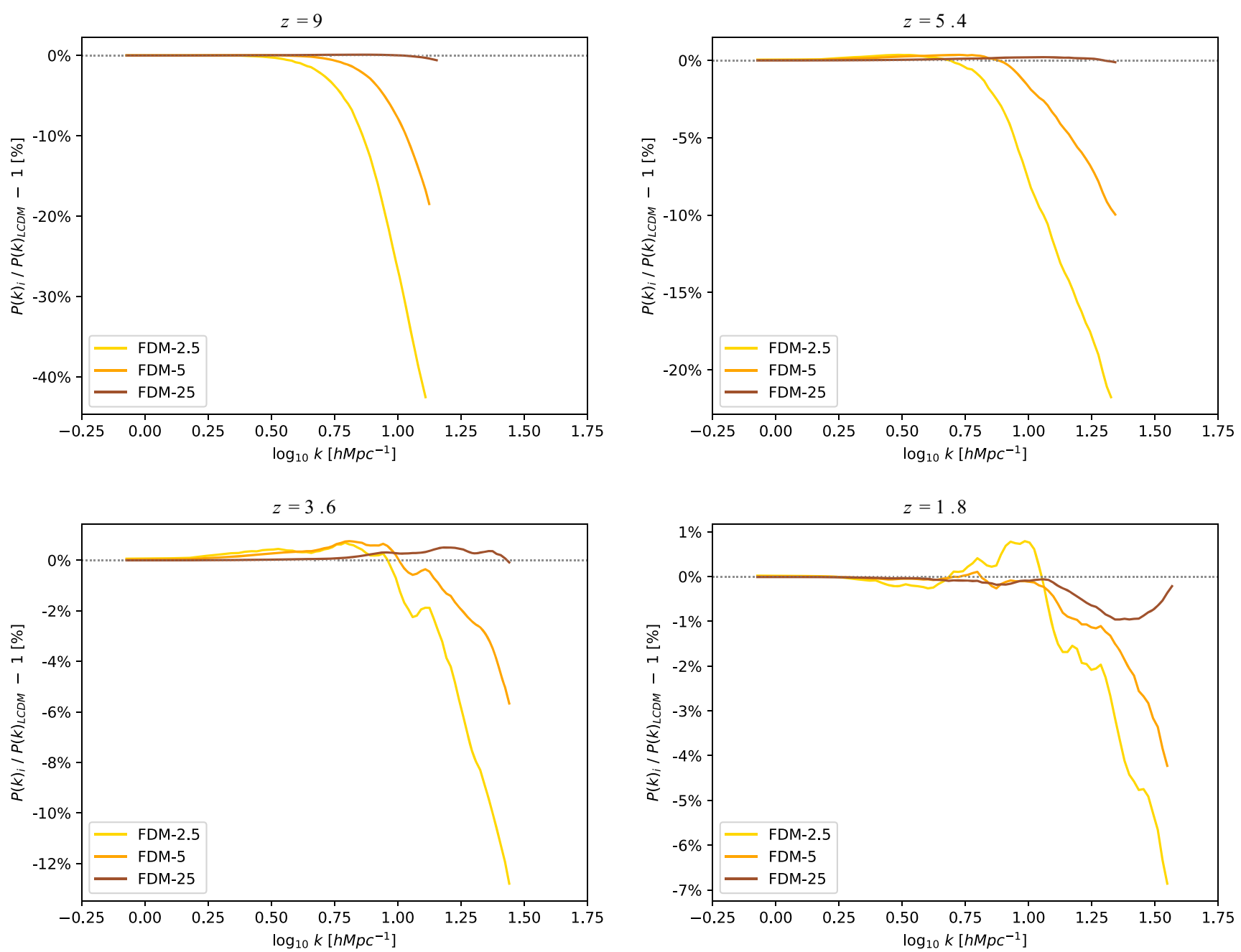

Figure 2. Matter power spectrum of FDM models contrasted with LCDM at different redshifts.

for the $m_{22}=2.5,5$, and $25 \mathrm{FDM}$ cases with negligible, between 1 and 2 per cent, differences between the FDMs and FDMnoQP cases.

In Fig. 4 we show the flux (top panel) and gas (bottom panel) $\mathrm{PDF}$ ratios between the simulations that include the QP and those that do not include it - FDM and FDMnoQP, respectively - at $z=$ 5.4 , one of the highest redshift bins in which Lyman $\alpha$ data are available.

It is possible to see that there is a $2-6$ per cent peak at flux $\sim 0.6-$ 0.8 , i.e. in regions of low transmissivity that are expected to trace voids. The fact that FDM simulations display a more peaked PDF compared to FDMnoQP ones for this range of fluxes means that, on average, in those models it is more likely to sample such void environments. In fact, the different PDFs should reflect the underlying different gas PDFs at the same redshifts and along the same lines of sight. In the bottom panel of Fig. 4, showing the corresponding gas PDF, it is indeed apparent that in models with FDMs the gas PDF is more skewed towards less dense regions, which are typically associated with high transmission. The effect due to the QP is thus to increase the volume-filling factor of regions below the mean density with respect to the corresponding FDMnoQP case.

In Fig. 5 we plot the percentage difference in terms of flux power spectrum at three different redshifts and for the FDM models, both compared to $\Lambda \mathrm{CDM}$ (right-hand panels) and to the corresponding
FDMnoQP case (left-hand panels). The increase of power at $z=$ 5.4 in the largest scales compared to the $\Lambda \mathrm{CDM}$ case is due to the imposed normalization at the same mean flux, while the evident suppression at small scales is related to the lack of structures at those scales. The comparison with the FDMnoQP set-ups, instead, reveals an additional suppression that is always below the 5 per cent level for all the masses considered. Since the flux power spectrum is an exponentially suppressed proxy of the underlying density field, these results are consistent with the matter power spectrum results previously shown in Figs 2 and 3.

Since the Lyman $\alpha$ constraints are calculated by weighting the contribution from all the scales, we expect the bound on $m_{22}$ found in Iršič et al. (2017a) to change comparably to the additional suppression introduced, which in our case is $2-3$ per cent.

This is exactly what can be seen in Fig. 6, where the marginalized posterior distribution of $m_{\chi}$ obtained in this work is plotted and compared with the results presented in Iršič et al. (2017a). The red line refers to our MCMC analysis, whereas the green line corresponds to the results obtained by Iršič et al. (2017a). The corresponding vertical lines show the $2 \sigma$ bounds on the FDM mass. The $2 \sigma$ bound on the FDM mass changes from $20.45 \times 10^{-22}$ to $21.08 \times 10^{-22} \mathrm{eV}$, which matches with our expectation and confirms that the approximation of neglecting the QP dynamical effects in Iršič et al. (2017a) was legitimate to investigate the Lyman $\alpha$ typ- 

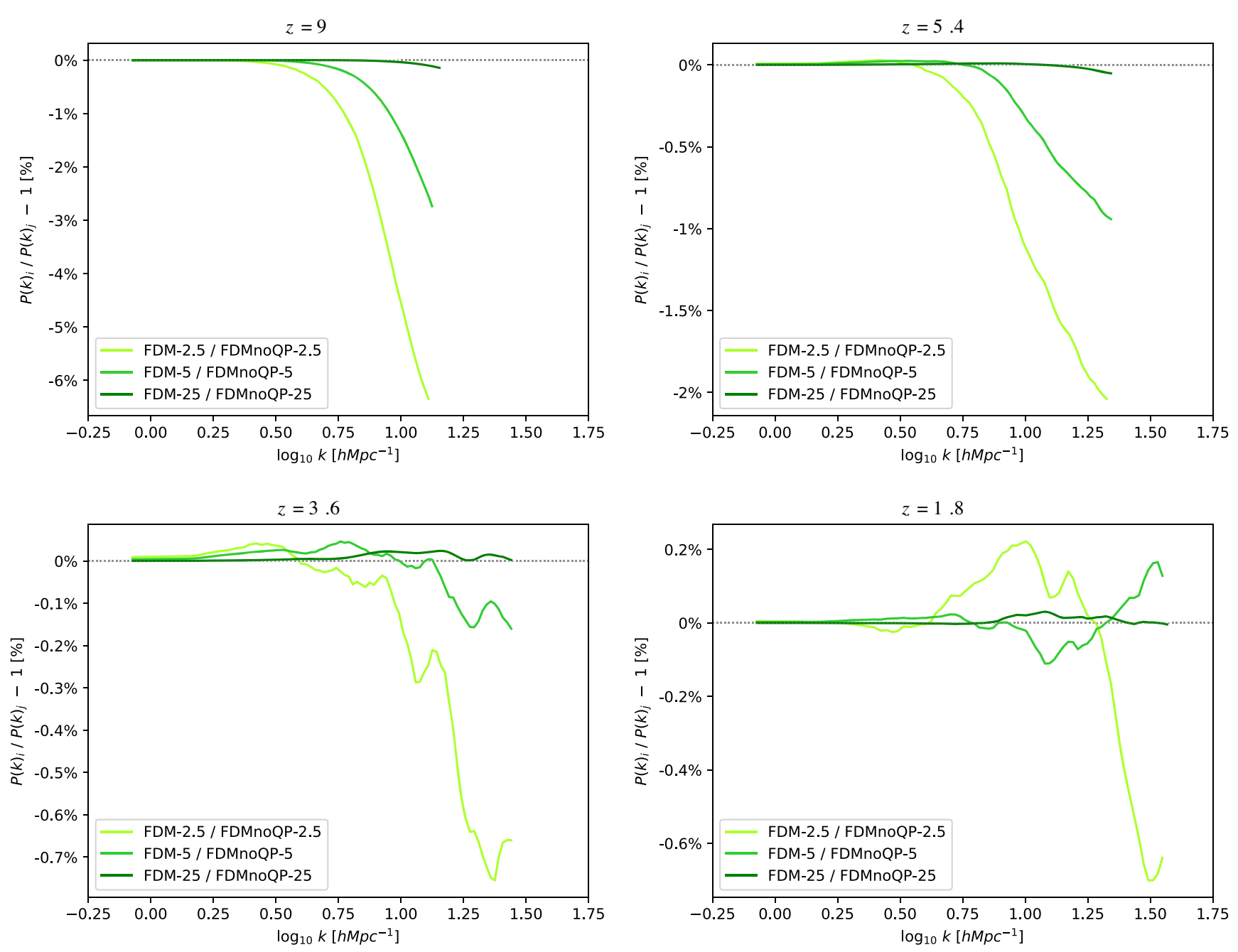

Figure 3. Matter power spectrum percentage differences between FDM simulation and their FDMnoQP counterpart at different redshifts.

ical scales. The agreement between the sets of results obtained with and without the dynamical QP implementation is evident and is not sensibly affected by varying the assumptions on the IGM thermal history.

This result represents to our knowledge the first FDM mass constraint derived from Lyman $\alpha$ forest observations that accounts for the full non-linear treatment of the QP, which introduces an additional albeit not big suppression of the matter power spectrum in the redshift range and comoving scales probed by the Lyman $\alpha$ forest. The agreement with previous results implies that the nonlinear evolution of the LSS and the non-linear mapping between flux and density effectively make up for the additional suppression introduced.

\subsection{Structure characterization}

The statistical properties of the genuine haloes belonging to each simulation are summarized in Fig. 7, where we display the cumulative halo mass function (top right panel), the halo mass outside $R_{200}$ (top left panel) where $R_{200}$ identifies the distance from the halo centre where the density is 200 times the critical density of the Universe and $M_{200}$ the mass contained within an $R_{200}$ radius sphere, the subhalo mass function (bottom left panel), and the subhalo radial distribution (bottom right panel). In order to highlight the impact of numerical fragmentation and simplify the comparison of the different models to $\Lambda \mathrm{CDM}$, relative ratios are displayed in the bottom panels and shaded lines represent the distribution of the full halo sample, i.e. including also spurious haloes.

The analytical fit used by Schive et al. (2016) to parametrize the cumulative HMF drop of the FDM models with respect to $\Lambda$ CDM

$N(>M)_{\mathrm{FDM}}=\int_{M}^{+\infty} \partial_{M} N_{\mathrm{CDM}}\left[1+\left(\frac{M}{M_{0}}\right)^{-1.1}\right]^{-2.2} \mathrm{~d} M$,

with $M_{0}=1.6 \times 10^{10} m_{22}^{-4 / 3} \mathrm{M}_{\odot}$, is plotted as reference - one for each FDM mass - in the top left panel of Fig. 7 (dotted lines).

As expected, we find that the number of small-mass subhaloes is drastically reduced in the FDM models and the cumulative distributions depart from $\Lambda \mathrm{CDM}$ at higher and higher masses as the $m_{\chi}$ mass decreases. The values at which the drop occurs are approximately $5 \times 10^{10}, 2.5 \times 10^{10}$, and $5 \times 10^{9} \mathrm{M}_{\odot}$ for values of $m_{22}$ of $2.5,5$, and 25 , respectively: this suggests a linear trend of the threshold mass

$M_{\mathrm{t}} \simeq 5 \times 10^{10} \mathrm{M}_{\odot}\left(\frac{2.5}{m_{22}}\right)$

describing the approximate mass below which the number of haloes starts decreasing with respect to $\Lambda \mathrm{CDM}$. 

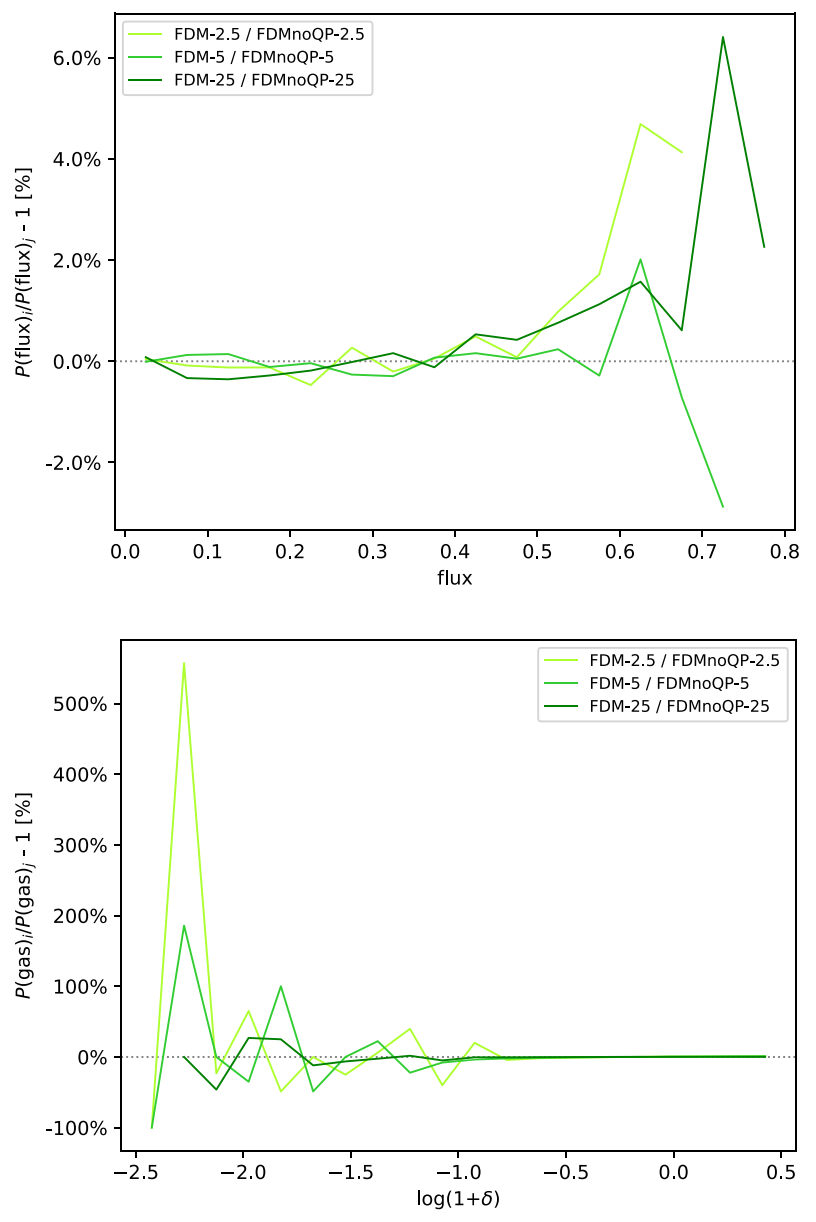

Figure 4. Relative differences of the flux PDF (top panel) and gas PDF (bottom panel) for FDM models with respect to their corresponding FDMnoQP counterparts, at redshift $z=5.4$.

Looking at the distribution of subhalo masses as compared to their associated primary halo $M_{200}$ and the radial distribution to $R_{200}$, it is evident how the numerous small subhaloes in $\Lambda \mathrm{CDM}$, far from the gravitational centre of the main halo, are the ones that were not able to form in an FDM universe.

The haloes that have masses above $M_{\mathrm{t}}$ not only have been able to survive the disrupting QP action up to redshift $z=0$, but the cumulative distribution shows how they also gained extra mass, at the smallest (sub)halo expenses. This is confirmed by the cumulative distribution of the primary structures $N\left(>M_{\mathrm{tot}}-M_{200}\right)$, representing the mass accumulated outside the $R_{200}$ radius, which is systematically higher with respect to $\Lambda \mathrm{CDM}$ case as the FDM mass lowers up to peaks of 200 per cent ratio for the lowest $m_{22}$ : this is consistent with the picture of bigger primary haloes accreting the mass of un-collapsed smaller subhaloes that did not form.

The fitting function of equation (21) is consistent with the scale of the drop of the HFM, which is indeed expected to be almost redshift independent, since it is predominantly given by the initial PS cut-off (Hu et al. 2000). However, it fails to reproduce the data on two levels: on the one hand, it does not recover the slope of the cumulative distribution - especially in the mass range close to $M_{\mathrm{t}}$ where the HMF departs from $\Lambda \mathrm{CDM}$ - and, on the other hand, it does not account for the mass transfer from smaller haloes, unable to collapse due to QP repulsive interaction, to bigger ones, which accrete the more abundant available matter from their surroundings. The discrepancies between the Schive et al. (2016) fitting function and our results are probably due to the fact that the former is based on simulations with approximated FDM dynamics and evolved only to redshifts $z=4$, thus representing a different collection of haloes that are, moreover, in an earlier stage of evolution. Therefore, the analysis of the aggregated data of cumulative distributions of genuine haloes in each simulation leads us to conclude that the formation, the evolution, and the properties of an FDM halo subject to the real effect of the QP as compared to the FDMnoQP approximation can follow three general paths depending on its own mass and on the mass of the FDM boson: if the halo mass is $M \ll M_{t}$, there is a high chance that the halo does not form at all since gravitational collapse is prevented by the $\mathrm{QP}$; if $M \gtrsim M_{\mathrm{t}}$, the halo can be massive enough to form but its properties will be affected by the QP - especially on its internal structure, as we will see below - while for $M \gg M_{\mathrm{t}}$ the halo is not severely affected by the QP, and will simply accrete more easily un-collapsed mass available in its surroundings.

In order to study in more detail the impact of FDM on the halo properties and structures, we divided our common sample, which by construction collects the haloes across all the simulations that share the same $\Lambda C D M$ match (as described in detail in Section 3.5), in three contiguous mass ranges. Let us remind that matching haloes have similar but not necessarily equal mass, so mass intervals are to be referred to the $\Lambda \mathrm{CDM}$ halo mass; the other matching haloes belonging to the FDM simulations are free to have lower and higher masses, compatibly with the limit imposed by the $\tilde{M}$ parameter of the common sample selection procedure. The common sample lowmass end is clearly limited by the FDM-2.5 model, since it is the one with higher $M_{\mathrm{t}}$, below which haloes have statistically lower chance to form. The three mass ranges are [0.5-4], [4-100], [100-4000] $\times$ $10^{10} \mathrm{M}_{\odot}$, in order to be compatible with the three halo categories described in the previous paragraph for the FDM-2.5 model, being $M_{\mathrm{t}}\left(m_{22}=2.5\right) \sim 5 \times 10^{10} \mathrm{M}_{\odot}$.

For all the matching haloes considered, we have tested the sphericity distribution, the halo volume and the total halo mass with respect to $\Lambda \mathrm{CDM}$, as well as the radial density profiles.

Properties of inter-simulation matching haloes are gathered in Fig. 8, where the total sample is divided column-wise in the three mass ranges. The sphericity, the volume occupied, and the total mass of the haloes contrasted with the corresponding $\Lambda$ CDM match are shown in the first row (left-hand panels), together with related distribution functions (right-hand panels). The second and third rows represent the overall density profiles, stacked in fractional spherical shells of $R_{200}$ and ellipsoidal shells of the major axis $a$ identified with the vertical dashed lines, respectively. Density profiles are divided by the value of the density calculated within the $R_{200}$ and $a$ shells and are shown both in absolute value (top panels) and relatively to $\Lambda \mathrm{CDM}$ (bottom panels).

The sphericity distributions confirm that, in the mass range considered, there is no statistical deviation from $\Lambda \mathrm{CDM}$, except for a mild deviation towards less spherical configurations of the less massive haloes, especially in the $m_{22}=2.5$ model. This is consistent with the analysis of the sphericity distributions of the genuine samples (see the lower panels in Fig. 1) that reveals that haloes appear to be statistically less spherical with respect to $\Lambda \mathrm{CDM}$ at $z=$ 0 when lower FDM masses are considered, down to a maximum of $\sim 10$ per cent decrease in sphericity for $m_{22}=2.5$ and a halo mass of $\sim 5 \times 10^{9} \mathrm{M}_{\odot}$.

For all the FDM models the volume occupied by the haloes is systematically larger, consistently with a delayed dynamical collapse of the haloes. All mass ranges show such property and it is emphasized by lower $m_{22}$ mass, i.e. stronger QP force; however, 


$$
z=5.4
$$
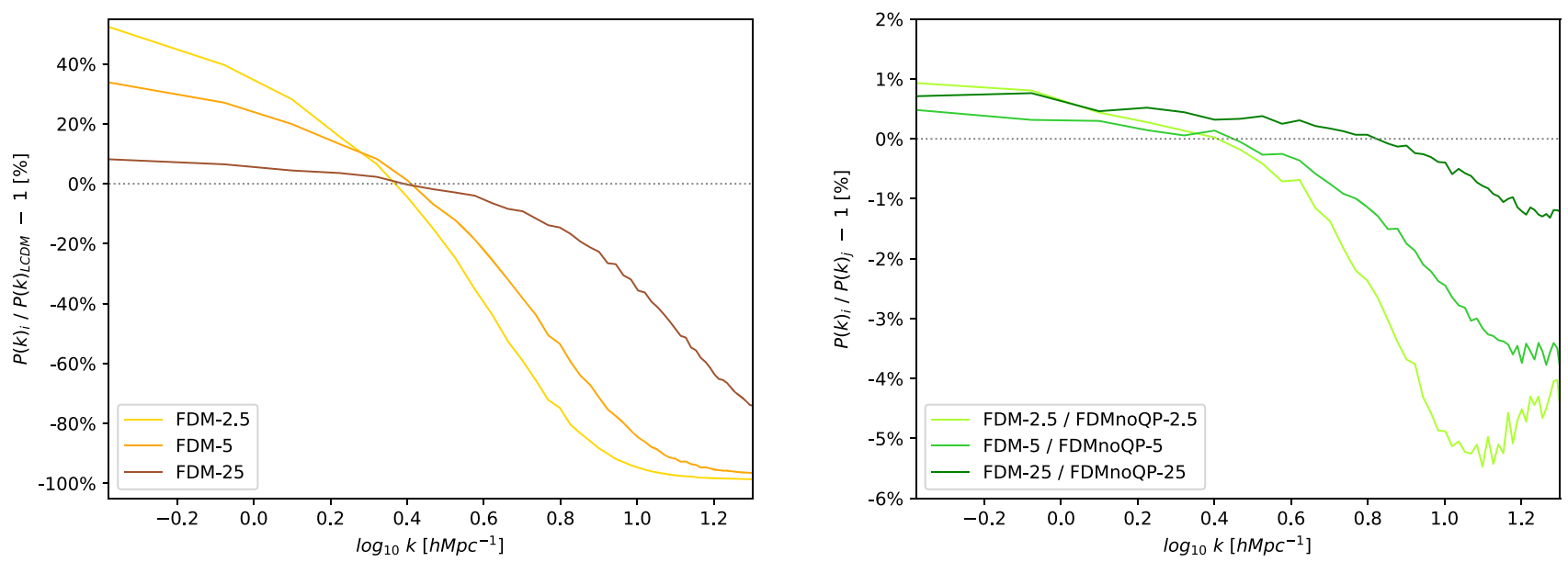

$$
z=4.0
$$
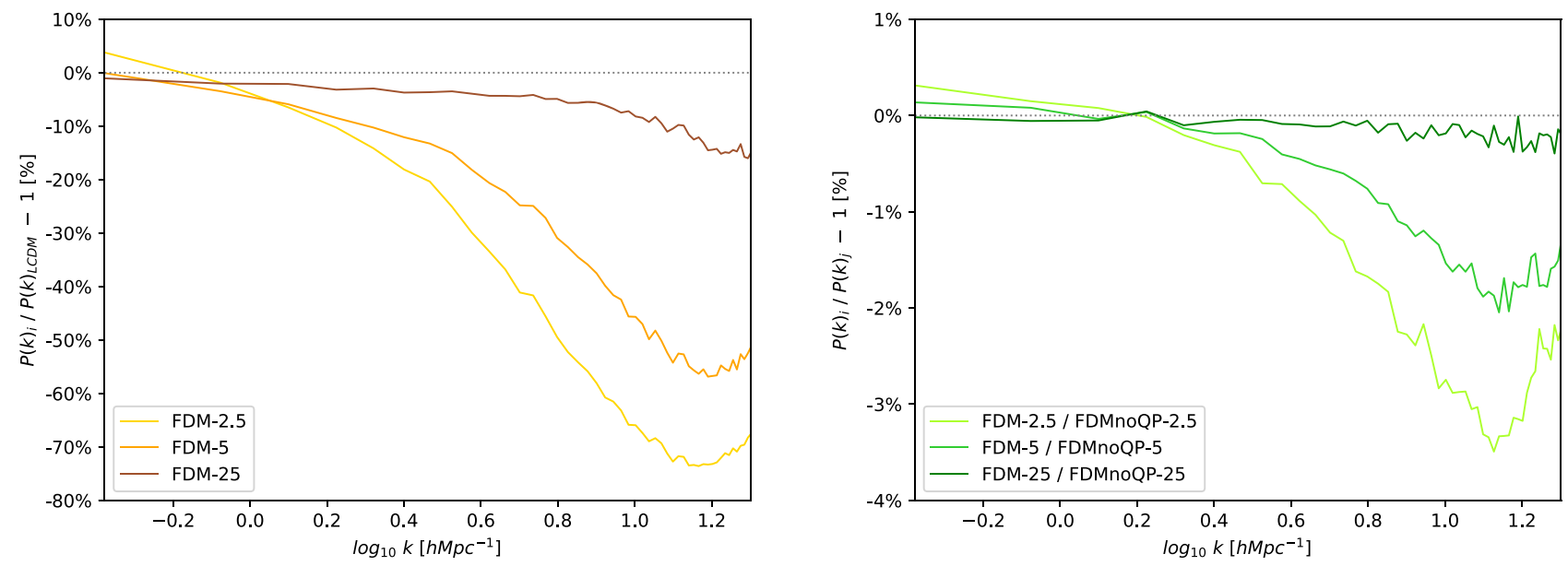

$$
z=3.0
$$
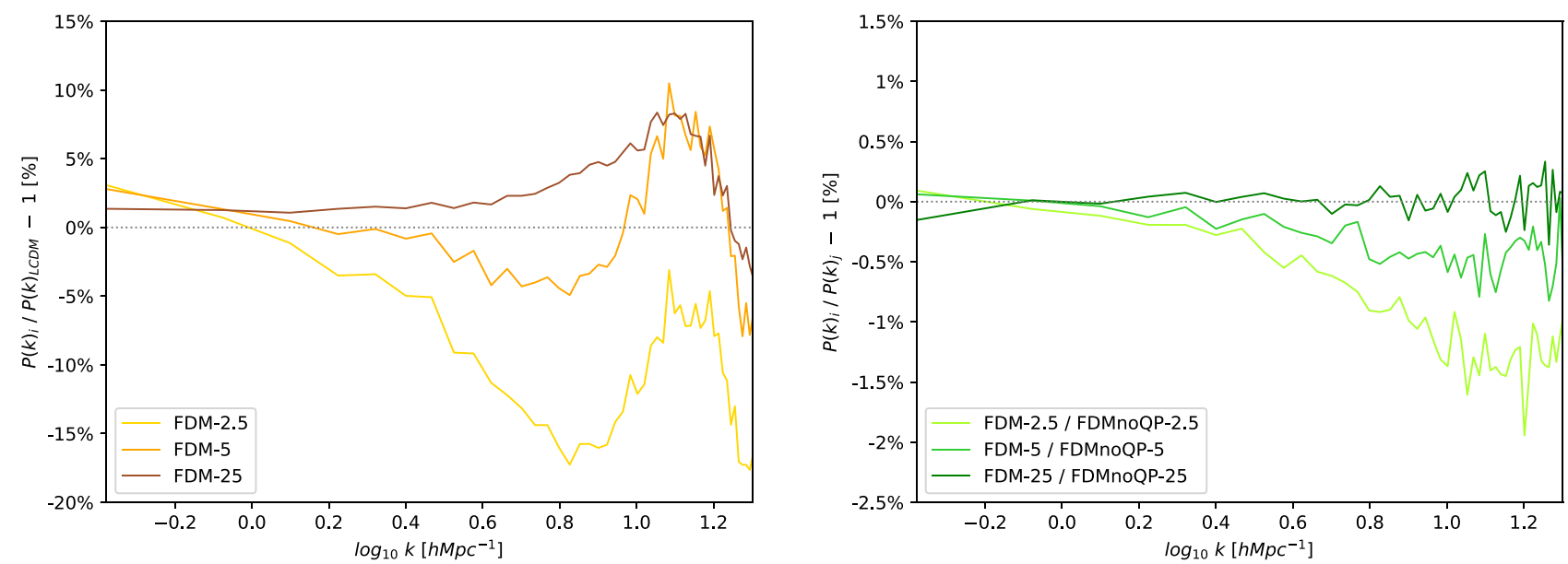

Figure 5. Flux power spectrum comparison between all simulations and LCDM (left-hand panels), and between FDM simulation and their FDMnoQP counterparts (right-hand panels) at different redshifts. 


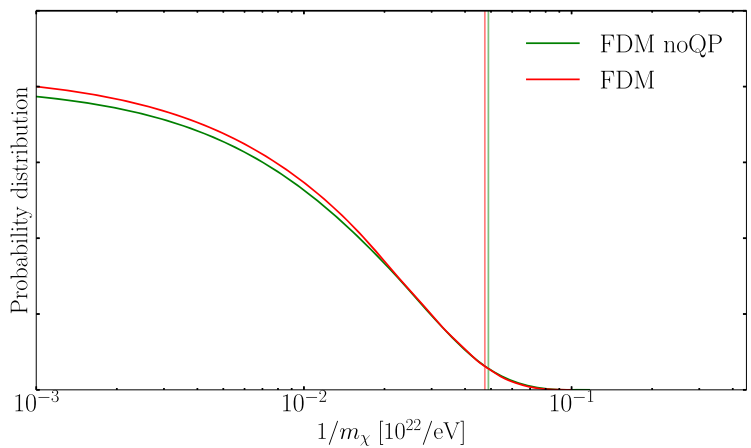

Figure 6. Here we plot the marginalized posterior distribution of $1 / m_{\chi}$ from both the analyses performed by Iršič et al. (2017a) (green lines, without QP) and ours (red lines, with QP). The vertical lines stand for the $2 \sigma$ C.L. limits.

while bigger haloes occupy almost systematically 20 per cent more volume for $m_{22}=2.5$, smaller haloes can reach even twice the volume occupied by their $\Lambda \mathrm{CDM}$ counterparts when the same model is considered.

Comparing the mass of the haloes in the various models with the one in $\Lambda \mathrm{CDM}$, it is possible to see that small haloes are less massive and big ones, on the contrary, become even more mas- sive, confirming our hypothesis of mass transfer from substructures towards main structures.

The stacked density profiles provide even more insight on the underlying different behaviour between the chosen mass ranges. Starting from the less massive one, the stacked profiles look very differently if plotted using the spherical $R_{200}$-based or the ellipsoidal $a$-based binning. This is due to two concurrent reasons related to the properties of this mass range: first of all, as we said before, the sphericity is $m_{\chi}$ dependent and thus it is not constant with respect to $\Lambda \mathrm{CDM}$, so the geometrical difference in the bin shape becomes important when different models are considered; secondly, since the FDM haloes have lower mass but occupy larger volumes, the two lengths are different from each other being $R_{200}$ related to density and $a$ purely to geometry so that the actual volume sampled is different. Nevertheless, it is possible to see that in FDM models there is an excess of mass in the outskirts of the halo seemingly peaking exactly at distance $a$ and less mass in the centre.

The intermediate-mass range shows also a suppression in the innermost regions but a less pronounced overdensity around $a$ as expected, since the effectiveness of the repulsive force induced by the QP in tilting the density distribution decreases as its typical scale becomes a smaller fraction of the size of the considered objects. In fact, stacked density profiles of the most massive haloes are very
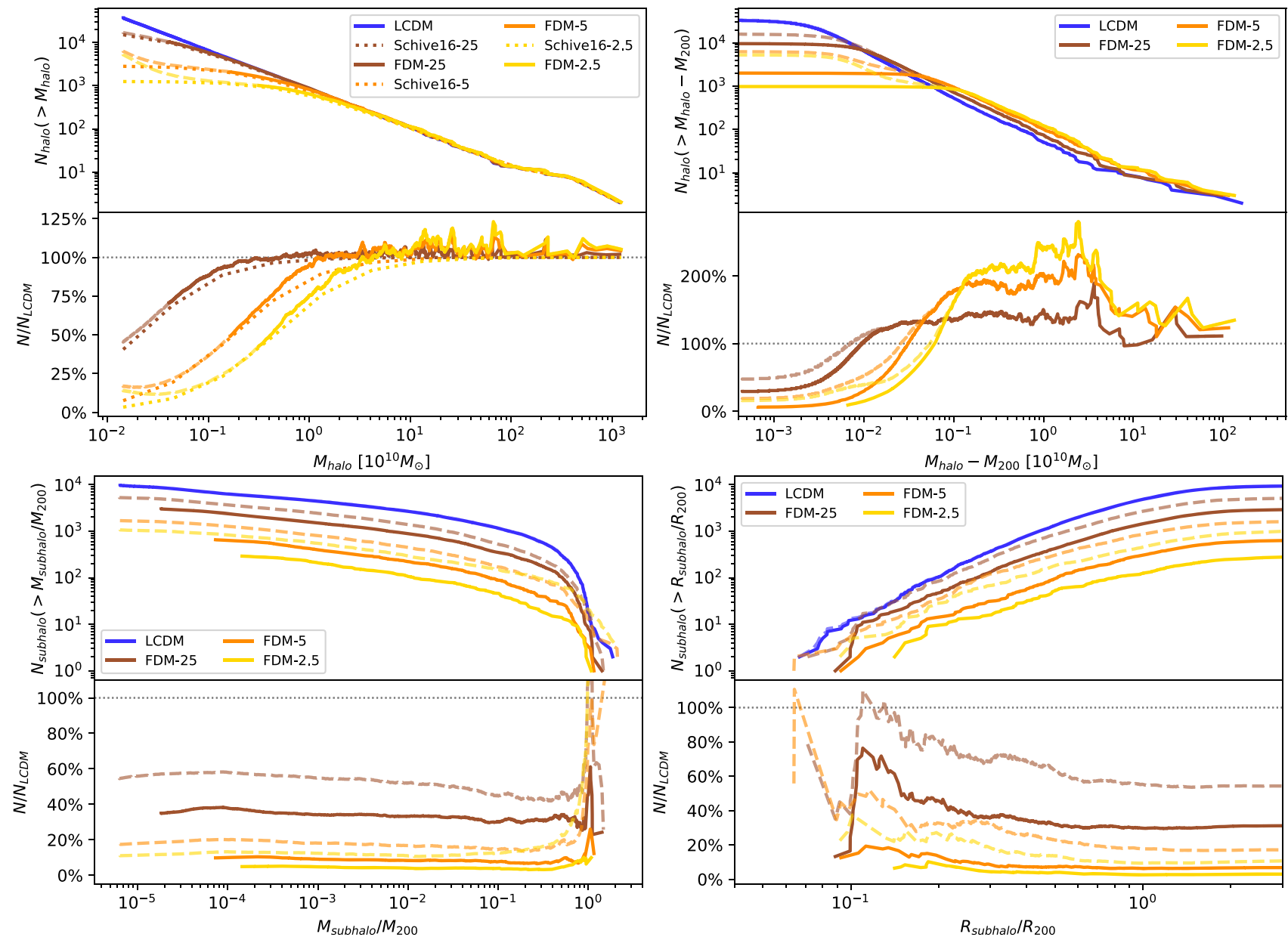

Figure 7. Properties of the halo and subhalo samples at $z=0$, with (dashed lines) and without (solid lines) including the haloes marked as spurious as described in Section 3.4. In particular, the cumulative distributions of halo mass (top left panel), the halo mass outside $R_{200}$ (top right panel), the subhalo-halo relative mass (bottom left panel), and the subhalo-halo distance (bottom right panel) are displayed. The fitting functions of the cumulative halo mass distribution of (Schive et al. 2016) of equation (21) are plotted for reference - the dotted line in the top left panel. 
$[0.5-4] \times 10^{10} M \odot$

- FDM-25 FDM-5 FDM-2.5
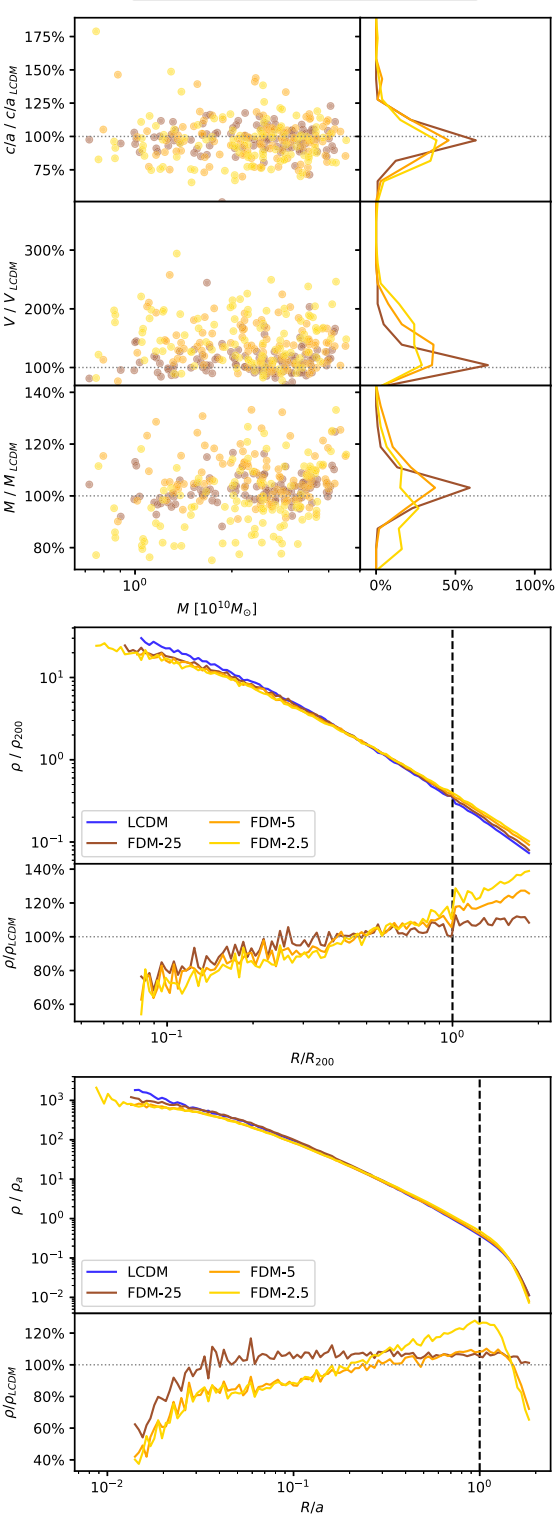

$[4-100] \times 10^{10} M \odot$
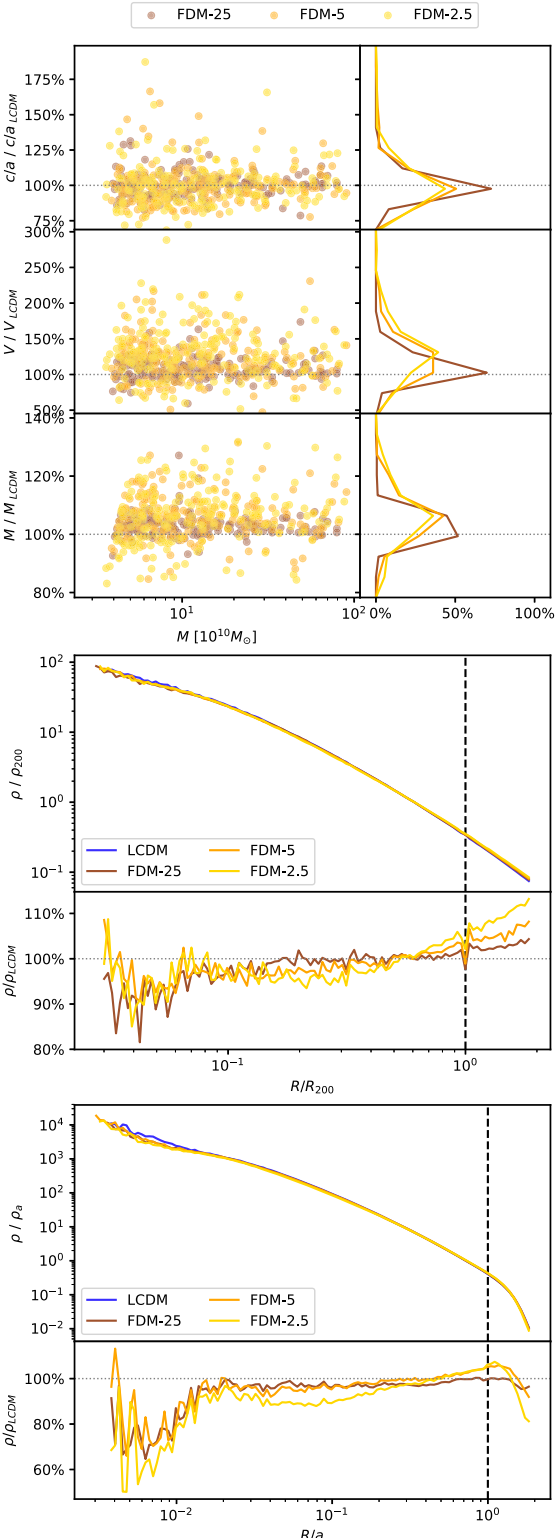

$[100-4000] \times 10^{10} M \odot$
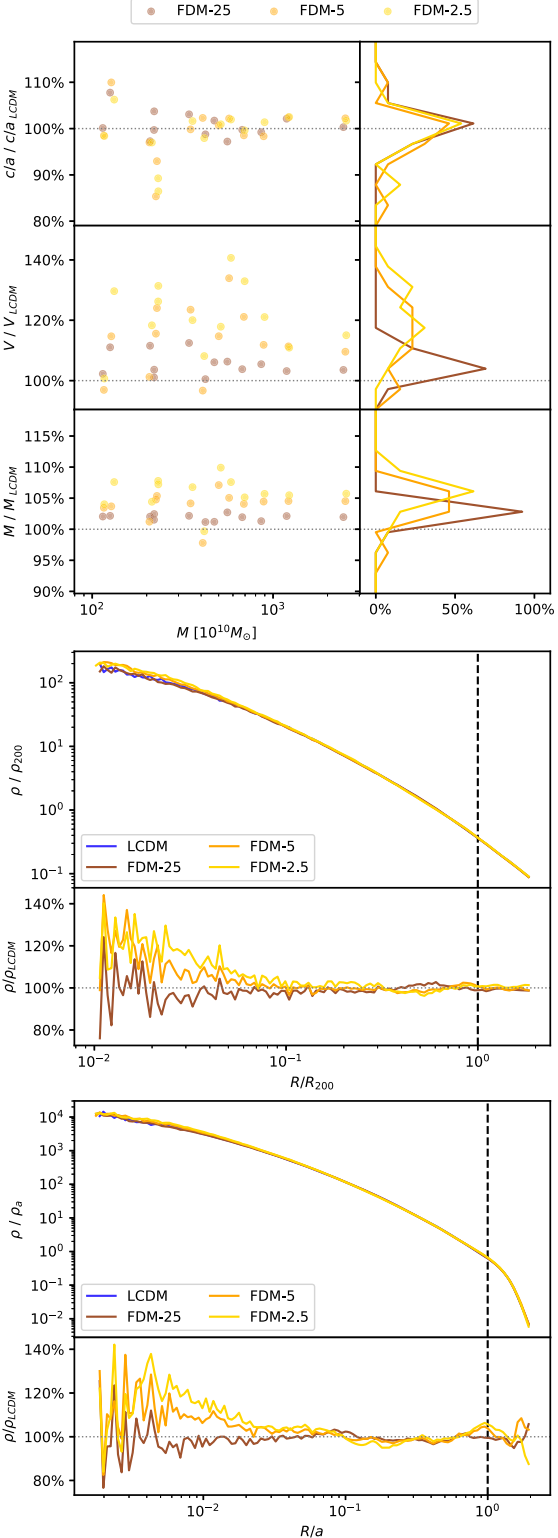

Figure 8. Properties of inter-simulation matching haloes. The total sample is divided column-wise in three mass ranges. The sphericity, the volume occupied, and the total mass of the haloes contrasted with the corresponding $\Lambda \mathrm{CDM}$ match are shown in the first row (left-hand panels), together with related distribution functions (right-hand panels). The second and third rows represent the overall density profiles, stacked in fractional spherical shells of $R_{200}$ and ellipsoidal shells of the major axis $a$ identified with the vertical dashed lines, respectively. Density profiles are divided by the value of the density calculated within $R_{200}$ and $a$ and are shown both in absolute value (top panels) and relatively to $\Lambda$ CDM. (bottom panels).

similar in the two binning strategies, being $R_{200} \sim a$ and sphericity constant among the various models, and consistent with no major deviation from $\Lambda \mathrm{CDM}$, except for a central overdensity. It is our opinion, however, that such a feature in the very centre of most massive haloes could be a numerical artefact, since its extension is comparable with the spatial resolution used.

The results presented in this section have been obtained through the detailed analysis of the statistical properties of haloes found at $z=0$ in the FDM simulations. The same analysis, repeated at $z=0$, of the FDMnoQP simulations shows very similar results that are, therefore, not shown in this work. Such consistency suggests that the properties of haloes at low redshift are at the investigated scales not sensible to modifications induced by the dynamical QP repulsive effect, which are expected to appear more prominently at scales of $\sim 1 \mathrm{Kpc}$ with the formation of solitonic cores.

\section{CONCLUSIONS}

We have presented the results obtained from two sets of numerical simulations performed with AX-GADGET, an extension of the massively parallel $N$-body code P-GADGET3 for non-linear simulations of FDM cosmologies, regarding Lyman $\alpha$ forest observations and the statistical detailed characterization of the LSSs. 
More specifically, our main aim was to design a set of simulations covering the typical scales and redshifts involved in Lyman $\alpha$ forest analyses, in order to extract synthetic observations, compare them with available Lyman $\alpha$ data, and finally to place a constraint on the mass of the FDM particle. In the literature, Lyman $\alpha$ forest was already used for this purpose but only in approximated set-ups, in which the quantum dynamical evolution of FDM was only encoded in the initial conditions transfer function, and neglected during the simulation (Armengaud et al. 2017; Kobayashi et al. 2017; Iršič et al. 2017a), while the AX-GADGET code allows us to drop such an approximation and take into account the non-linear effects of full FDM dynamics.

The constrain on the FDM mass we find is $21.08 \times 10^{-22} \mathrm{eV}$, which is 3 per cent higher with respect to what was found in Iršič et al. (2017a). The fact that these two bounds are similar, despite the different dynamical evolution considered in these different works, implies that the additional suppression deriving from the QP dynamical contribution, at the scales and redshifts probed by Lyman $\alpha$, is compensated by the gravitational growth of perturbations when these enter the non-linear regime, implying also that even if the QP does play a role in the LSS evolution the approximation of Iršič et al. (2017a) (also adopted by Armengaud et al. 2017; Kobayashi et al. 2017) is valid and sufficient at these scales.

Secondly, we studied in detail the statistical properties of the LSSs through the analysis of the aggregated data on haloes regarding their mass, volumes, and shapes, as well as their individual inner structure.

The main results regarding the effects of FDM on LSS that we found can be summarized as follows:

(i) the FDM particle mass $m_{22}$ defines a typical mass scale $M_{\mathrm{t}} \simeq$ $1.25 \times 10^{11} / m_{22} \mathrm{M}_{\odot}$ characterizing the halo distribution of different FDM models; all halo properties can be interpreted within the framework of having two families of haloes: the small ones with $M \lesssim M_{\mathrm{t}}$ and the big ones with $M \gg M_{\mathrm{t}}$ (since the very small ones $M \ll M_{\mathrm{t}}$ do not form at all);

(ii) small haloes, according to the above definition, show outward tilted profiles and a lower total mass, and are less spherical and more voluminous, so less dense overall;

(iii) big haloes instead are almost unaffected in their internal structure - apart from the expected solitonic inner cores that we cannot resolve with our simulations -. They occupy a larger volume and they also have higher total mass, mostly collected outside $R_{200}$, compatibly with the accretion of a fraction of the subhalo mass, which was not able to collapse into substructures due to the QP repulsive interaction.

To conclude, we have performed for the first time a suite of hydrodynamical simulations of a statistically significant volume of the Universe for FDM models featuring a fully consistent implementation of the QP effects on the dynamical evolution of the system. These simulations allowed us to perform for the first time a fully consistent comparison of mock Lyman $\alpha$ observations with available data and to update existing constraints on the allowed FDM mass range. As the new constraints are not significantly different from previous ones, this represents the first direct validation of the approximations adopted in previous works. Furthermore, our large halo sample allowed us to perform an extensive characterization of the properties of dark matter haloes in the context of FDM scenarios, highlighting the typical mass scale below which FDM effects start to appear. Higher resolution simulations will soon allow us to explore even smaller scales where we expect to observe the formation of solitonic cores.

\section{ACKNOWLEDGEMENTS}

$\mathrm{MN}$ and MB acknowledge support from the Italian Ministry for Education, University and Research (MIUR) through the Scientific Independence of young Researchers (SIR) individual grant SIMCODE, project number RBSI14P4IH. The simulations described in this work have been performed on the Marconi supercomputer at Consorzio interuniversitario per il calcolo automatico (CINECA) thanks to the Partnership for Advanced Computing in Europe (PRACE) allocation 2016153604. MV and RM are supported by INFN I.S. PD51-INDARK. VI is supported by US NSF grant AST1514734.

\section{REFERENCES}

Abbott B. P. et al., 2017, Phys. Rev. Lett., 119, 161101

Alam S. et al., 2017, MNRAS, 470, 2617

Albert A. et al., 2017, ApJ, 834, 110

Angulo R. E., Hahn O., Abel T., 2013, MNRAS, 434, 3337

Arinyo-i Prats A., Miralda-Escudé J., Viel M., Cen R., 2015, JCAP, 1512, 017

Armengaud E., Palanque-Delabrouille N., Marsh D. J. E., Baur J., Yèche C., 2017, MNRAS, 471, 4606

Bahcall N. A., Fan X.-h., 1998, ApJ, 504, 1

Baldi M., Pettorino V., Robbers G., Springel V., 2010, MNRAS, 403, 1684

Bekenstein J. D., 2004, Phys. Rev. D, 70, 083509

Bel J. et al., 2014, A\&A, 563, A37

Bertone G., Hooper D., Silk J., 2005, Phys. Rep., 405, 279

Bode P., Ostriker J. P., Turok N., 2001, ApJ, 556, 93

Bolton J. S., Puchwein E., Sijacki D., Haehnelt M. G., Kim T.-S., Meiksin A., Regan J. A., Viel M., 2017, MNRAS, 464, 897

Bosma A., 1981, AJ, 86, 1825

Boylan-Kolchin M., Bullock J. S., Kaplinghat M., 2012, MNRAS, 422, 1203

Brookshaw L., 1985, Proceedings of the Astronomical Society of Australia, 6. 207

Brooks A. M., Kuhlen M., Zolotov A., Hooper D., 2013, ApJ, 765, 22

Buonaura A. 2018, Proc. Sci., Searches for Direct Pair Production of Third Generation Squarks with the ATLAS Detector. SISSA, Trieste, (PoS\#079),

Chavanis P. H., 2012, A\&A, 537, A127

Chesler P. M., Loeb A., 2017, Phys. Rev. Lett., 119, 031102

Cleary P. W., Monaghan J. J., 1999, J Comput. Phys., 148, 227

Clowe D., Bradac M., Gonzalez A. H., Markevitch M., Randall S. W., Jones C., Zaritsky D., 2006, ApJ, 648, L109

Colin F., Egli R., Lin F. Y., 2006, J. Comput. Phys., 217, 680

Crocce M., Pueblas S., Scoccimarro R., 2006, MNRAS, 373, 369

Danninger M., 2017, J. Phys. Conf. Ser., 888, 012039

Davis M., Efstathiou G., Frenk C. S., White S. D., 1985, ApJ, 292, 371

De Martino I., Broadhurst T., Tye S. H. H., Chiueh T., Schive H.-Y., 2018, preprint (arXiv:1807.08153)

Heymans C. et al., 2013, MNRAS, 432, 2433

Hildebrandt H. et al., 2017, MNRAS, 465, 1454

Hlozek R., Grin D., Marsh D. J. E., Ferreira P. G., 2015, Phys. Rev. D, 91, 103512

Hu W., Barkana R., Gruzinov A., 2000, Phys. Rev. Lett., 85, 1158

Hui L., Gnedin N. Y., 1997, MNRAS, 292, 27

Hui L., Ostriker J. P., Tremaine S., Witten E., 2017, Phys. Rev. D, 95, 043541 Iršič V., Viel M., Haehnelt M. G., Bolton J. S., Becker G. D., 2017a, Phys. Rev. Lett., 119, 031302

Iršič V. et al., 2017b, MNRAS, 466, 4332

Iršič V. et al., 2017c, Phys. Rev. D, 96, 023522

Kaplinghat M., Knox L., Turner M. S., 2000, Phys. Rev. Lett., 85, 3335

Kashlinsky A., 1998, Phys. Rep., 307, 67

Klypin A. A., Kravtsov A. V., Valenzuela O., Prada F., 1999, ApJ, 522, 82

Kobayashi T., Murgia R., De Simone A., Iršiš V., Viel M., 2017, Phys. Rev.

D, 96, 123514

Komatsu E. et al., 2011, ApJS, 192, 18 
Koopmans L. V. E., Treu T., 2003, ApJ, 583, 606

Lin S.-C., Schive H.-Y., Wong S.-K., Chiueh T., 2018, Phys. Rev. D, 97, 103523

López S. et al., 2016, A\&A, 594, A91

Lovell M. R., Frenk C. S., Eke V. R., Jenkins A., Gao L., Theuns T., 2014, MNRAS, 439, 300

Maccio A. V., Paduroiu S., Anderhalden D., Schneider A., Moore B., 2012, MNRAS, 424, 1105

Madelung E., 1927, Z. Phys., 40, 322

Marsh D. J. E., 2016a, preprint (arXiv:1605.05973)

Marsh D. J. E., 2016b, Phys. Rep., 643, 1

Marsh D. J. E., Ferreira P. G., 2010, Phys. Rev. D, 82, 103528

Marsh D. J. E., Pop A.-R., 2015, MNRAS, 451, 2479

Mateo M. L., 1998, ARA\&A, 36, 435

McDonald P. et al., 2006, ApJS, 163, 80

Medvedev M. V., 2014, Phys. Rev. Lett., 113, 071303

Melott A. L., Shandarin S. F., 1989, ApJ, 343, 26

Milgrom M., 1983, ApJ, 270, 365

Mocz P., Succi S., 2015, Phys. Rev. E, 91, 053304

Mocz P., Lancaster L., Fialkov A., Becerra F., 2018, Phys. Rev. D, 97, 083519

Murgia R., Iršič V., Viel M., 2018, Phys. Rev. D, 98, 083540

Nori M., Baldi M., 2018, MNRAS, 478, 3935

Oh S.-H., de Blok W. J. G., Brinks E., Walter F., Kennicutt R. C., Jr., 2011, AJ, 141, 193

Oman K. A. et al., 2015, MNRAS, 452, 3650

Peebles P. J. E., 1980, The Large-scale Structure of the Universe. Princeton Univ. Press, Princeton, NJ

Persic M., Salucci P., Stel F., 1996, MNRAS, 281, 27

Planck Collaboration XV, 2015, A\&A, 594, A15

Planck Collaboration XIII, 2016, A\&A, 594, A13

Puchwein E., Baldi M., Springel V., 2013, MNRAS, 436, 348

Rocha M., Peter A. H. G., Bullock J. S., Kaplinghat M., Garrison-Kimmel S., Onorbe J., Moustakas L. A., 2013, MNRAS, 430, 81

Rubin V. C., Ford W. K., Thonnard N., 1980, ApJ, 238, 471

Sanders R. H., McGaugh S. S., 2002, ARA\&A, 40, 263

Schive H.-Y., Tsai Y.-C., Chiueh T., 2010, ApJS, 186, 457

Schive H.-Y., Chiueh T., Broadhurst T., 2014, Nature Phys., 10, 496

Schive H.-Y., Chiueh T., Broadhurst T., Huang K.-W., 2016, ApJ, 818, 89

Schive H.-Y., ZuHone J. A., Goldbaum N. J., Turk M. J., Gaspari M., Cheng C.-Y., 2018, MNRAS, 481, 4815

Schneider A., Smith R. E., Maccio A. V., Moore B., 2012, MNRAS, 424, 684

Seljak U., Slosar A., McDonald P., 2006, JCAP, 0610, 014

Spergel D. N., Steinhardt P. J., 2000, Phys. Rev. Lett., 84, 3760

Springel V., 2005, MNRAS, 364, 1105

Springel V., White S. D. M., Tormen G., Kauffmann G., 2001, MNRAS, 328,726

Towler M. D., Russell N. J., Valentini A., 2011, Proceedings of the Royal Society A: Mathematical, Physical and Engineering Sciences, 468, 990

Veltmaat J., Niemeyer J. C., Schwabe B., 2018, Phys. Rev. D, 98, 043509

Viel M., Haehnelt M. G., Springel V., 2004, MNRAS, 354, 684

Viel M., Lesgourgues J., Haehnelt M. G., Matarrese S., Riotto A., 2005, Phys. Rev. D, 71, 1

Viel M., Becker G. D., Bolton J. S., Haehnelt M. G., 2013, Phys. Rev. D, 88,043502

Wang J., White S. D. M., 2007, MNRAS, 380, 93

Woo T.-P., Chiueh T., 2009, ApJ, 697, 850

Zwicky F., 1937, ApJ, 86, 217

\section{APPENDIX A: NOTE ON DE BROGLIE-BOHM INTERPRETATION}

In the de Broglie-Bohm (DBB) interpretation of quantum mechanics, the Universe possesses at each time a well-defined configuration that evolves under the influence of the wavefunction of the system, also known as 'pilot wave'. For simplicity and analogy with our problem, let us reduce the Universe configuration to the collective position of $\mathrm{N}$ boson particles: in this case the configuration $Q \equiv\left(\mathbf{q}_{1}, \mathbf{q}_{2}, \ldots, \mathbf{q}_{N}\right) \in \mathbb{R}^{3 N}$ is physically related to the quantum wavefunction $\hat{\phi}(Q, t) \in \mathbb{C}^{3 N}$.

The wavefunction that governs the evolution of $Q$ is the so-called 'guiding function'

$\frac{\mathrm{d}}{\mathrm{d} t} \mathbf{q}_{k}=\frac{\hbar}{m_{k}} \Im \frac{\nabla_{k} \hat{\phi}(Q)}{\hat{\phi}(Q)}$

while the pilot wave evolves under the standard Schrödinger equation

$i \hbar \partial_{t} \hat{\phi}(Q)=-\sum_{k=1}^{N} \frac{\hbar^{2}}{2 m_{\chi}} \nabla_{k}^{2} \hat{\phi}(Q)+V \hat{\phi}(Q)$.

The DBB is explicitly non-local, given the dependence of velocity of a single particle $k$ on the global wavefunction that represents the whole particle ensemble configuration. The Bose-Einstein condensate assumption is then a key ingredient to recover locality that is intimately connected with SPH and its approximation of neighbours-only cut-off. Under condensation, the wavefunction factorizes

$\hat{\phi}(Q) \equiv \hat{\phi}\left(q_{1}, q_{2}, \ldots, q_{N}\right)=\prod_{k=1}^{N} \hat{\phi}_{k}\left(q_{k}\right)$

and, consequently, equation (A1) reduces to equation (3) where the dependence on all the other particles different from $k$ cancels in the ratio.

In the DBB framework, the Born Rule $\rho=|\hat{\phi}|^{2}$ is neither assumed nor even imposed, allowing for quantum non-equilibrium states for which this condition is not fulfilled. Yet, it has been shown numerically how a system in which the Born Rule is not initially verified eventually evolves towards quantum equilibrium $\rho \rightarrow|\hat{\phi}|^{2}$ and, once reached, does not leave (Towler, Russell \& Valentini 2011).

Therefore, our answer to the fair question on the consistency of representing a non-local quantum interaction as QP with particle ensembles is that the SPH description of a bosonic FDM species in the Bose-Einstein condensation regime is theoretically robust and coherent with wave-based portrayals. In Nori \& Baldi (2018) we showed how SPH is indeed able to recover some of the FDM results obtained with full-wave solvers.

It is nevertheless important to stress that the equivalence between the Eulerian and Lagrangian pictures does not ensure overlapping results in terms of numerical simulation, since the intrinsic temporal and spatial resolution is finite and affects the two differently. For these reasons, however, it is our belief that incompatibilities between the two approaches are to ascribe only to resolution limits.

In this sense it is very interesting the convergence to the classical results in the limit $\hbar / m \rightarrow 0$ shown in Mocz et al. (2018), where the potential and the force in the Schrödinger-Poisson description obtained by simulations in several tests approach the classical Vlasov-Poisson ones while the density field is however unable to do the same due to uncontrollable interference patterns.

Studying the accuracy and the behaviour in limit cases of numerical realizations of quantum systems not only is necessary to estimate the deviation between simulations and observations but can be useful to improve our understanding of statistical representations of quantum nature objects.

This paper has been typeset from a $\mathrm{T}_{\mathrm{E}} \mathrm{X} / \mathrm{LAT} \mathrm{E}$ file prepared by the author. 